The Astrophysical Journal, 632:638-658, 2005 October 10

(C) 2005. The American Astronomical Society. All rights reserved. Printed in U.S.A.

\title{
FIVE NEW MULTICOMPONENT PLANETARY SYSTEMS ${ }^{1}$
}

\author{
Steven S. Vogt, ${ }^{2}$ R. Paul Butler,${ }^{3}$ Geoffrey W. Marcy, ${ }^{4}$ Debra A. Fischer, ${ }^{4,5}$ Gregory W. Henry, ${ }^{6}$ \\ Greg Laughlin, ${ }^{2} \mathrm{~J}_{\text {ASON T. Wright, }}{ }^{4}$ and John A. Johnson ${ }^{4}$ \\ Received 2005 January 20; accepted 2005 June 22
}

\begin{abstract}
We report Doppler measurements for six nearby G- and K-type main-sequence stars that show multiple low-mass companions, at least one of which has planetary mass. One system has three planets, the fourth triple-planet system known around a normal star, and another has an extremely low minimum mass of $18 M_{\oplus}$. HD 128311 (K0 V) has two planets (one previously known) with minimum masses $(M \sin i)$ of $2.18 M_{\mathrm{J}}$ and $3.21 M_{\mathrm{J}}$ and orbital periods of 1.26 and $2.54 \mathrm{yr}$, suggesting a possible $2: 1$ resonance. For HD $108874(\mathrm{G} 5 \mathrm{~V})$, the velocities reveal two planets (one previously known) having minimum masses and periods of $\left(M \sin i_{b}=1.36 M_{\mathrm{J}}, P_{b}=1.08 \mathrm{yr}\right)$ and $\left(M \sin i_{c}=\right.$ $\left.1.02 M_{\mathrm{J}}, P_{c}=4.4 \mathrm{yr}\right)$. HD $50499(\mathrm{G} 1 \mathrm{~V})$ has a planet with $P=6.8 \mathrm{yr}$ and $M \sin i=1.7 M_{\mathrm{J}}$, and the velocity residuals exhibit a trend of $-4.8 \mathrm{~m} \mathrm{~s}^{-1} \mathrm{yr}^{-1}$, indicating a more distant companion with $P>10 \mathrm{yr}$ and minimum mass of $2 M_{\mathrm{J}}$. HD 37124 (G4 IV-V) has three planets, one having $M \sin i=0.61 M_{\mathrm{J}}$ and $P=154.5$ days, as previously known. We find two plausible triple-planet models that fit the data, both having a second planet near $P=840$ days, with the more likely model having its third planet in a $6 \mathrm{yr}$ orbit and the other one in a 29 day orbit. For HD 190360, we confirm the planet having $P=7.9 \mathrm{yr}$ and $M \sin i=1.5 M_{\mathrm{J}}$ as found by the Geneva team, but we find a distinctly noncircular orbit with $e=0.36 \pm 0.03$, rendering this not an analog of Jupiter as had been reported. Our velocities also reveal a second planet with $P=17.1$ days and $M \sin i=18.1 M_{\oplus}$. HD 217107 (G8 IV) has a previously known "hot Jupiter" with $M \sin i=1.4 M_{\mathrm{J}}$ and $P=7.13$ days, and we confirm its high eccentricity, $e=0.13$. The velocity residuals reveal an outer companion in an eccentric orbit, having minimum mass of $M \sin i>2 M_{\mathrm{J}}$, eccentricity $e \sim 0.5$, and a period $P>8 \mathrm{yr}$, implying a semimajor axis $a>4$ AU and providing an opportunity for direct detection. We have obtained high-precision photometry of five of the six planetary host stars with three of the automated telescopes at Fairborn Observatory. We can rule out significant brightness variations in phase with the radial velocities in most cases, thus supporting planetary reflex motion as the cause of the velocity variations. Transits are ruled out to very shallow limits for HD 217107 and are also shown to be unlikely for the prospective inner planets of the HD 37124 and HD 108874 systems. HD 128311 is photometrically variable with an amplitude of $0.03 \mathrm{mag}$ and a period of 11.53 days, which is much shorter than the orbital periods of its two planetary companions. This rotation period explains the origin of periodic velocity residuals to the two-planet model of this star. All of the planetary systems here would be further constrained with astrometry by the Space Interferometry Mission.
\end{abstract}

Subject headings: planetary systems — stars: individual (HD 128311, HD 108874, HD 217107, HD 50499, HD 37124, HD 190360)

\section{INTRODUCTION}

Systems of multiple planets found by the Doppler technique have provided numerous clues about the formation, dynamics, and nature of exoplanets in general. The first system of planets found around a main-sequence star, $v$ Andromedae (Butler et al. 1999), offered the first comparison of another planetary system

\footnotetext{
${ }^{1}$ Based on observations obtained at the W. M. Keck Observatory, which is operated jointly by the University of California and the California Institute of Technology. Keck time has been granted by both NASA and the University of California.

${ }^{2}$ University of California Observatories/Lick Observatory, University of California, 373 Interdisciplinary Sciences, Santa Cruz, CA 95064; vogt@ucolick.org.

3 Department of Terrestrial Magnetism, Carnegie Institution of Washington, 5241 Broad Branch Road NW, Washington, DC 20015-1305.

4 Department of Astronomy, University of California, 601 Campbell Hall, Berkeley, CA 94720-3411.

5 Department of Physics and Astronomy, San Francisco State University, 1600 Holloway Avenue, San Francisco, CA 94132.

${ }^{6}$ Center of Excellence in Information Systems, Tennessee State University, 330 10th Avenue North, Nashville, TN 37203; and Senior Research Associate, Department of Physics and Astronomy, Vanderbilt University, Nashville, TN 37235 .
}

to our solar system. It also provided the first association of the odd characteristics of single exoplanets (close-in "hot" Jupiters and eccentric orbits) to planetary systems in general. The previously detected single objects of Jupiter mass (e.g., Mayor \& Queloz 1995; Butler \& Marcy 1996; Marcy \& Butler 1996) had left open the possibility of a nonplanetary nature for them.

Indeed, $v$ Andromedae foreshadowed the diagnostic value of multiplanet systems in several ways. Upper limits to the orbiting masses of $\sim 20 M_{\mathrm{J}}$ were provided both by the dynamical stability of the system (Rivera \& Lissauer 2000; Lissauer \& Rivera 2001; Chiang et al. 2001) and by the upper limits to any astrometric wobble (ESA 1997; Butler et al. 1999). The system of three planets orbiting a central star distinguished it structurally from hierarchical multiple-star systems, suggesting formation in a protoplanetary disk (Lissauer 1995; Levison et al. 1998). Moreover, the close-in planet and two outer eccentric orbits in the $v$ And system conferred planetary status to those single exoplanets that resided in similar, but unanticipated, orbits.

New multiplanet systems are beginning to offer further clues about planetary formation and dynamics. To date, 14 multiplanet systems are known around both main-sequence and subgiant stars (Marcy et al. 2004; Mayor et al. 2004), not including those 
TABLE 1

Stellar Properties

\begin{tabular}{|c|c|c|c|c|c|c|c|c|c|}
\hline HD & HIP & Spectral Type & $\begin{array}{c}\pi \\
\text { (mas) }\end{array}$ & $\begin{array}{c}M_{V} \\
\text { (mag) }\end{array}$ & $\begin{array}{l}M_{\text {star }} \\
\left(M_{\odot}\right)\end{array}$ & $\log R_{\mathrm{HK}}^{\prime}$ & $\begin{array}{c}\text { Jitter } \\
\left(\mathrm{m} \mathrm{s}^{-1}\right)\end{array}$ & $\begin{array}{c}P_{\text {rot }} \\
\text { (days) }\end{array}$ & $\log ($ age $/ y r)$ \\
\hline $37124 \ldots \ldots \ldots$ & 26381 & G4 IV-V & 30.1 & 5.07 & 0.78 & -4.90 & 3.2 & 25 & 9.59 \\
\hline 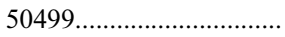 & 32970 & G1 V & 21.2 & 3.85 & 1.24 & -5.02 & 2.8 & 21 & 9.79 \\
\hline 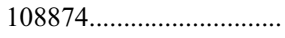 & 61028 & G5 V & 14.6 & 4.58 & 0.99 & -5.07 & 3.9 & 38 & 9.85 \\
\hline $128311 \ldots \ldots \ldots \ldots \ldots \ldots$ & 71395 & $\mathrm{~K} 0 \mathrm{~V}$ & 60.4 & 6.41 & 0.84 & -4.4 & 8.9 & 14 & $<9.3^{\mathrm{a}}$ \\
\hline 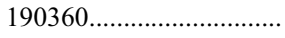 & 98767 & G6 IV & 62.9 & 4.70 & 0.96 & -5.09 & 3.1 & 40 & 9.89 \\
\hline $217107 \ldots \ldots \ldots \ldots \ldots \ldots \ldots \ldots \ldots \ldots \ldots \ldots \ldots \ldots \ldots$ & 113421 & G8 IV & 50.7 & 4.71 & 1.05 & -5.08 & 3.4 & 39 & 9.87 \\
\hline
\end{tabular}

${ }^{\text {a }}$ Parameter $\log R_{\mathrm{HK}}^{\prime}$ indicates activity and youth, but $P_{\mathrm{rot}}$ and age are poorly determined; beyond domain of Ca II $\mathrm{H}$ and $\mathrm{K}$ calibration.

reported here. Gravitational interactions among the planets in multiplanet systems shape the final configuration of the system and shed light on the dissipative medium when the interactions occurred (Bryden et al. 2000; Rivera \& Lissauer 2001; Laughlin \& Chambers 2001; Lee \& Peale 2002; Chiang \& Murray 2002; Ford et al. 2003; Ida \& Lin 2004). Interactions between pairs of planets may result in orbital resonances and pumping of orbital eccentricities (Nelson \& Papaloizou 2002; Nauenberg 2002; Marzari \& Weidenschilling 2002; Goździewski 2003). Subsequent eccentricity damping in the protoplanetary disk may also occur. The distribution of final orbital sizes may result from inward migration and mutual interactions among the planets, in a race against the dissipation of the protoplanetary disk, leaving the planets frozen in their tracks (Armitage et al. 2003; Trilling et al. 2002; D'Angelo et al. 2003; Thommes \& Lissauer 2003; Ida \& Lin 2004; Alibert et al. 2005).

Yet, the paucity of known multiplanet systems prevents us from distinguishing the dominant, common processes from the stochastic rarities. The discovery and characterization of more multiple planetary systems will help reveal key processes that shape systems in general and put our solar system in context. Here we present five new planetary systems containing one planet and at least one additional companion, most of which are likely to be planetary. We further update another such system, HD 217107.

\section{THE TARGET STARS AND DOPPLER TECHNIQUE}

The complete target list of 975 FGKM main-sequence stars and subgiants is provided in Wright et al. (2004) and Valenti $\&$ Fischer (2005), which also contain their measured surface properties, ages, and metallicities. The selection criteria for the target stars were described in Marcy et al. (2005). The stars were drawn from the Hipparcos catalog (ESA 1997) with criteria that they have $B-V>0.55$, have decl. $>-35^{\circ}$, be older than $2 \mathrm{Gyr}$, and reside within $3 \mathrm{mag}$ of the main sequence. In roughly four equal bins of $B-V$, the brightest available such stars were chosen. We measure $\mathrm{Ca}$ II $\mathrm{H}$ and $\mathrm{K}$ emission lines to estimate the age and rotation of the stars (Wright et al. 2004; Noyes et al. 1984), and we carry out LTE spectrum synthesis to derive $T_{\text {eff }}$, $\log g$, and $[\mathrm{Fe} / \mathrm{H}]$ for all stars (Valenti \& Fischer 2005; Fischer \& Valenti 2005).

We estimate the mass of each target star by linearly interpolating within a grid of model stellar evolution calculations computed by Girardi et al. (2002), by using $M_{V}$ and $(B-V)$ values of each star found by Hipparcos (ESA 1997). We use metallicities from Valenti \& Fischer (2005) to choose the specific model grid. The resulting stellar masses are accurate to within $\sim 10 \%$, with errors accumulating from both the observed uncertain stellar parameters (distance, luminosity) and uncertainties in the models. See Fernandes \& Santos (2004) regarding possible
$10 \%$ errors in mass from such models due to mixing-length uncertainties. Our final masses disagree with those listed by Allende Prieto \& Lambert (1999) by typically less than $0.1 M_{\odot}$, with differences presumably due to their adoption of solar metallicities.

Table 1 lists the derived properties of the six stars considered here. The first and second columns identify the stars with HD and Hipparcos names, respectively. The third column gives the spectral type and luminosity class as listed in SIMBAD, the fourth column gives the parallax from Hipparcos, and the fifth column gives the resulting absolute visual magnitude, $M_{V}$. The sixth column gives the mass of the star, as determined above from the Girardi et al. (2002) models. The seventh column gives the logarithm of the fraction of the stellar luminosity that emerges as chromospheric emission at the $\mathrm{Ca}$ II $\mathrm{H}$ and $\mathrm{K}$ lines, namely, $\log R_{\mathrm{HK}}^{\prime}$, and the eighth column gives the resulting photospheric jitter in $\mathrm{m} \mathrm{s}^{-1}$, estimated as described in Marcy et al. (2005) and Wright et al. (2005). The ninth and tenth columns give the expected rotation period and age of the star based on $R_{\mathrm{HK}}^{\prime}$ from Wright et al. (2004) and Noyes et al. (1984). Figure 1 shows the $\mathrm{Ca}$ II $\mathrm{H}$ spectral line for all stars discussed in this paper. The emission reversal is apparent at the core of the line of some of the stars, an indication of magnetic heating of chromospheric gas.

We began the Doppler monitoring of most stars at the Keck 1 telescope in 1997 or 1998. All Doppler shift measurements were made using our iodine cell technique in which a glass cell filled with iodine molecules at a pressure of $0.01 \mathrm{~atm}$ and temperature of $50^{\circ} \mathrm{C}$ is placed just ahead of the focal plane of the telescope (Marcy \& Butler 1992; Butler 1993; Butler et al. 1996). Most of the observations herein were made with the Keck 1 telescope and HIRES, but some (for HD 217107) were also made with the Lick Observatory $3 \mathrm{~m}$ telescope and the Hamilton spectrometer (Vogt 1987; Vogt et al. 1994). Figure 2 shows velocity measurements for representative stars on our Keck planet search that exhibit no radial velocity variation. The typical rms of 3$4 \mathrm{~m} \mathrm{~s}^{-1}$ is representative of our errors plus the photospheric jitter of such stars.

The six stars presented here have spectral types from G1 to $\mathrm{K} 0$, all located either on the main sequence or within 1 mag of it (see Table 1). Their $V$ magnitudes range from $V=6.2$ to 8.8 , corresponding to exposure times on the Keck telescope of 1-8 minutes, respectively, in typical seeing and sky conditions. The resulting signal-to-noise ratio of the spectrum per pixel was typically 300 , with each pixel spanning a wavelength range corresponding to $\lambda / \Delta \lambda=143,000$. The spectrometer point-spread function (PSF) has FWHM $=2.4$ pixels $(24 \mu \mathrm{m}$ in size) with our slit width of 0.86 , yielding a resolution of $R=60,000$. Systematic errors of $2-3 \mathrm{~m} \mathrm{~s}^{-1}$ were caused by subtle charge transfer efficiency nonlinearities of the Tektronix CCD used for most observations here. Including all errors, both photon limited and 


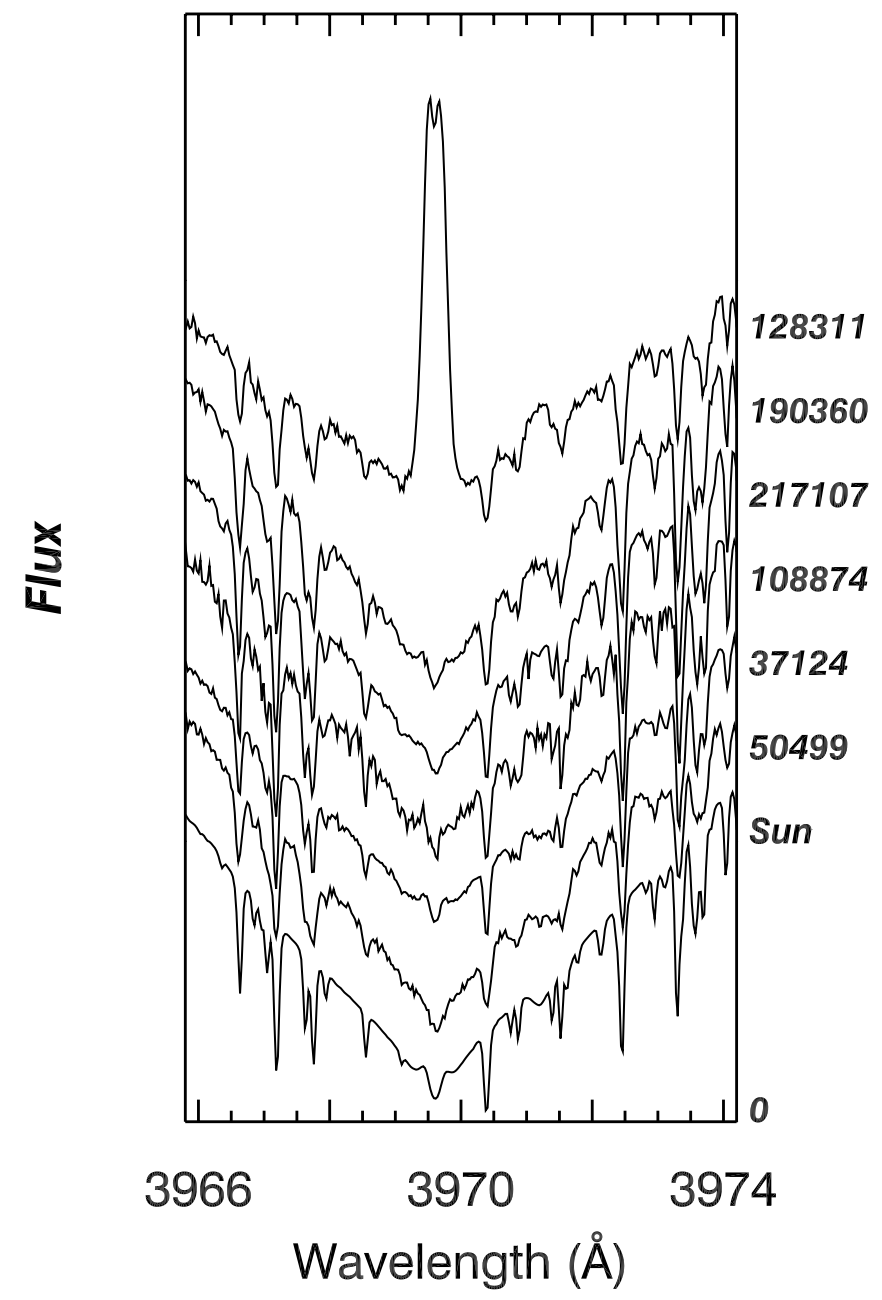

FIg. 1.- Spectra near the $\mathrm{Ca}$ II $\mathrm{H}$ line for all six stars discussed here. The emission reversals reflect magnetic activity on stars that correlates with photospheric velocity jitter. The chromospheric emission, measured as a fraction of stellar luminosity, $\log R_{\mathrm{HK}}^{\prime}$, is listed in Table 1 , along with the inferred values of jitter.

systematic, we achieve a Doppler precision of $2.5-4 \mathrm{~m} \mathrm{~s}^{-1}$, depending on rotational $V \sin i$. In 2004 August, we began using a new CCD made by MIT Lincoln Labs, with $15 \mu \mathrm{m}$ pixels and superior charge transfer characteristics. At most a few observations have been made with this new detector. Representative "null" stars that show no Doppler variation above those errors are presented in many past papers such as Marcy et al. (2005), and additional null stars are provided here in Figure 2.

Stars of spectral type FGK exhibit intrinsic velocity "jitter" due to acoustic $p$-modes, turbulent convection, starspots, and flows in magnetic regions. We predict the jitter for each of the six stars presented here from the empirical velocity behavior of the ensemble of hundreds of similar stars in the same domain of the H-R diagram and from their chromospheric emission (Saar et al. 1998; Cumming et al. 1999; Santos et al. 2000a; Wright et al. 2005). Some of the jitter is no doubt actually instrumental, stemming from small errors in the data analysis at levels of $1-2 \mathrm{~m} \mathrm{~s}^{-1}$. Predicted jitter values for each star are listed in Table 1 .

The fitting of orbital models is carried out by assigning to each Doppler measurement a weight, $w_{i}$, that is the inverse of the quadrature sum of the internal velocity uncertainty, $\epsilon_{i}$, and the jitter, $w_{i}=1 /\left(\epsilon_{i}^{2}+\right.$ jitter $\left.^{2}\right)$. The orbital parameters from those fits are presented in Table 2. For each star presented here (except HD 50499), we fit the sum of two Keplerian orbits to the

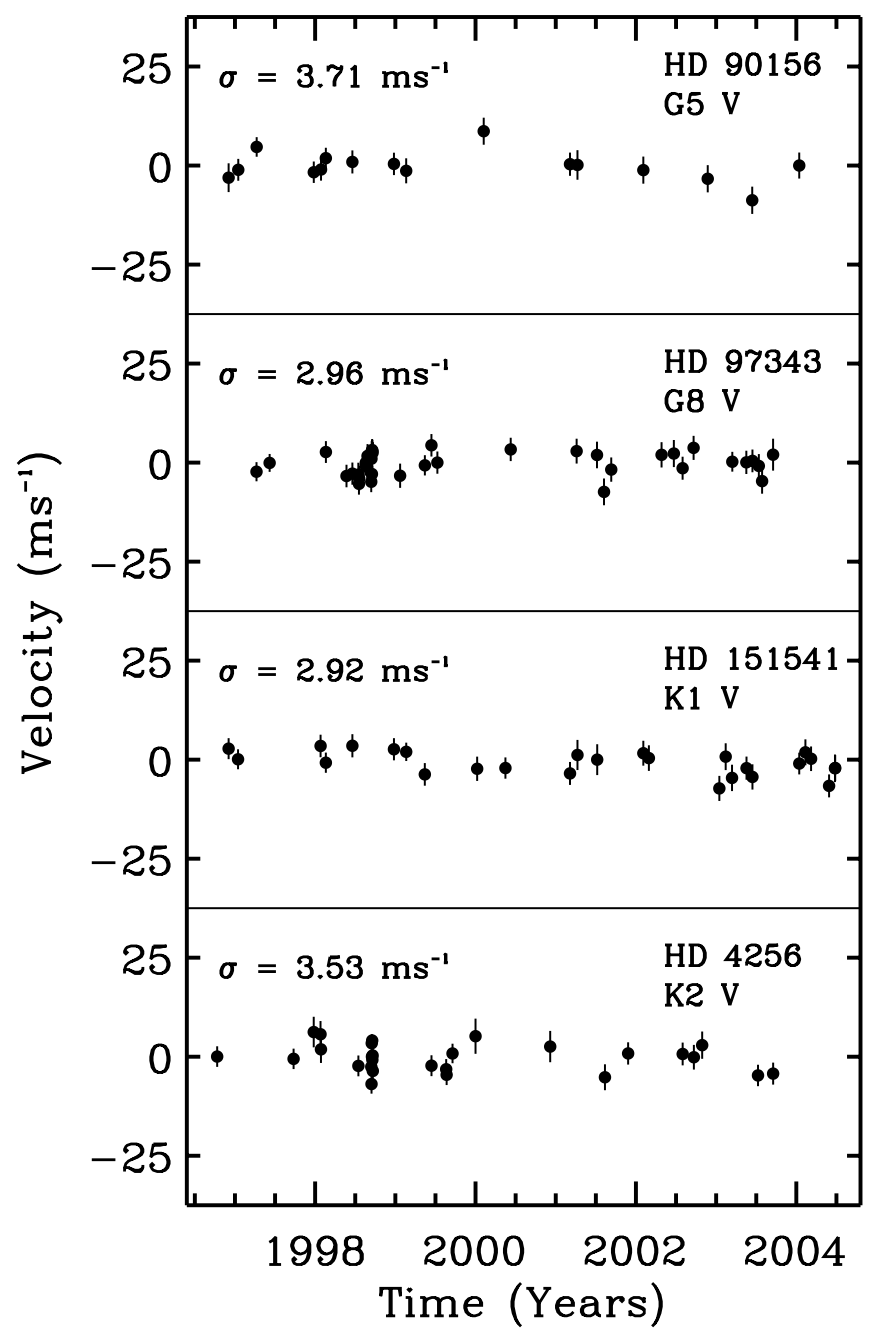

FIG. 2.-Measured velocities vs. time for four representative stars that show no significant velocity variation during $6 \mathrm{yr}$ of observations. The standard deviation of velocities ranges from 2.9 to $3.7 \mathrm{~m} \mathrm{~s}^{-1}$, which includes both the errors and photospheric jitter. The four stars, HD 90158, HD 97343, HD 151541, and HD 4256, span the range of spectral types and brightnesses of the candidate planet-bearing stars discussed here, suggesting that they represent empirically the errors and jitter of those stars.

measured velocities. We ignore gravitational interactions between the planets. For several stars, notably HD 128311, mutual gravitational perturbations may affect somewhat the optimal characterization of the system. Such $N$-body calculations are beyond the scope of this paper but will be described in a later paper by G. Laughlin et al. (2005, in preparation). Nonetheless, the noninteracting Keplerian models presented here provide a necessary starting point for the searches within the multiparameter space of dynamical calculations.

We determine the uncertainties in the orbital parameters by determining the best-fit double-Keplerian model and subtracting that theoretical velocity curve from the measured velocities. The resulting residuals are scrambled, keeping the times of observation the same. We then construct 100 realizations of the velocities, each set consisting of the best-fit theoretical velocity curve added to scrambled residuals. For each realization, we determine the best-fit Keplerian orbit, and we quote the standard deviation of the resulting set of orbital parameters as the " $1 \sigma$ " uncertainty. This approach may underestimate the true uncertainties, notably of the eccentricity, especially for fits that are poorly constrained. For systems such as HD 217107, a 
TABLE 2

Orbital Parameters

\begin{tabular}{|c|c|c|c|c|c|c|c|}
\hline $\begin{array}{l}\text { Planet } \\
\text { (b or c) }\end{array}$ & $\begin{array}{l}\text { Period } \\
\text { (days) }\end{array}$ & $\begin{array}{c}T_{p} \\
(\mathrm{JD}-2,440,000)\end{array}$ & $e$ & $\begin{array}{c}\omega \\
(\operatorname{deg})\end{array}$ & $\begin{array}{c}K \\
\left(\mathrm{~m} \mathrm{~s}^{-1}\right)\end{array}$ & $\begin{array}{c}M \sin i \\
\left(M_{\mathrm{J}}\right)\end{array}$ & $\begin{array}{c}a \\
(\mathrm{AU})\end{array}$ \\
\hline HD $37124 b^{\mathrm{a}} \ldots \ldots \ldots \ldots \ldots$ & 154.46 & 10000.11 & 0.055 & 140.5 & 27.5 & 0.61 & 0.53 \\
\hline 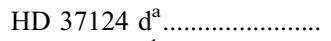 & 2295 & 9606 & 0.2 (fix) & 266 & 12.2 & 0.66 & 3.19 \\
\hline HD $37124 b^{b} \ldots \ldots \ldots \ldots$ & 154.70 & 9970.1 & 0.25 & 76.8 & 30.3 & 0.66 & 0.52 \\
\hline HD $37124 c^{b}$. & 839.6 & 9603.1 & 0.15 & 27.3 & 18.7 & 0.73 & 1.60 \\
\hline 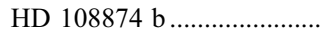 & $395.4(2.5)$ & $10131.5(60)$ & $0.07(0.04)$ & $248.4(36)$ & $38.0(2.5)$ & $1.36(0.13)$ & $1.051(0.02)$ \\
\hline HD 108874 c ……… & $1605.8(88)$ & $9584.8(180)$ & $0.25(0.07)$ & $17.3(23)$ & $18.4(1.6)$ & $1.018(0.3)$ & $2.68(0.25)$ \\
\hline HD 128311 b ......................... & $458.6(6.8)$ & $10210.9(76)$ & $0.25(0.10)$ & $110.9(36)$ & $66.8(8.7)$ & $2.18(0.22)$ & $1.099(0.04)$ \\
\hline 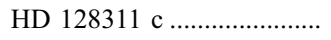 & $928.3(18)$ & $10012.2(401)$ & $0.17(0.09)$ & $195.5(150)$ & $76.2(4.6)$ & $3.21(0.30)$ & $1.76(0.13)$ \\
\hline 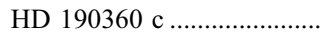 & $17.10(0.015)$ & $10000.07(0.9)$ & $0.01(0.1)$ & $153.7(32)$ & $4.6(1.1)$ & $0.057(0.015)$ & $0.128(0.002)$ \\
\hline 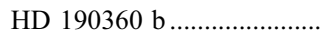 & $2891(85)$ & $10628.1(99.8)$ & $0.36(0.03)$ & $12.4(9.3)$ & $23.5(0.5)$ & $1.502(0.13)$ & $3.92(0.2)$ \\
\hline HD 217107 b ......................... & $7.1269(0.00022)$ & $9998.4241(0.17)$ & $0.13(0.02)$ & $21.1(7.6)$ & $140.7(2.6)$ & $1.37(0.14)$ & $0.074(0.002)$ \\
\hline
\end{tabular}

${ }^{\mathrm{a}}$ Model A: $\mathrm{rms}=4.14,\left(\chi_{\nu}^{2}\right)^{1 / 2}=0.96$.

${ }^{\mathrm{b}}$ Model B: $\mathrm{rms}=5.12,\left(\chi_{\nu}^{2}\right)^{1 / 2}=1.14$.

${ }^{c}$ Fit includes a linear slope of $-4.8 \mathrm{~m} \mathrm{~s}^{-1} \mathrm{yr}^{-1}$ due to another companion.

${ }^{\mathrm{d}}$ Fit from the Keck Observatory alone.

${ }^{\mathrm{e}}$ Fit from Lick Observatory alone.

Bayesian Monte Carlo analysis would provide superior estimation of the orbital element uncertainties for the long-period outer companion (see, e.g., Ford 2004).

\section{VELOCITIES AND ORBITAL PARAMETERS FOR THE SIX STARS}

\subsection{HD 128311}

Based on our earlier Keck HIRES Doppler measurements of HD 128311 (HIP 71395) obtained from 1998-2002, we reported evidence for a planet having orbital period $P=422$ days, eccentricity $e=0.31$, and semiamplitude $K=85 \mathrm{~m} \mathrm{~s}^{-1}$, implying a minimum mass of $M \sin i=2.6 M_{\mathrm{J}}$ (Butler et al. 2003). The Keplerian model included an apparent linear trend of $30 \mathrm{~m} \mathrm{~s}^{-1}$ $\mathrm{yr}^{-1}$, suggesting existence of another companion orbiting farther from the star. With another $2 \mathrm{yr}$ accumulated to date, the trend has proceeded to reveal curvature and periodicity, apparently caused by an additional orbiting companion. The new orbital analysis of this two-component system also benefits from updated measurements of the stellar parameters, notably the mass and photospheric velocity jitter.

HD 128311 has a spectral type K0 V, parallax of 60.4 mas (ESA 1997), and $M_{V}=6.41$, residing on the main sequence (see Table 1). It has a metallicity of $0.00 \pm 0.03 \mathrm{dex}$ (Valenti \& Fischer 2005). Its $\mathrm{Ca}$ II $\mathrm{H}$ and $\mathrm{K}$ lines show strong emission reversals in our HIRES spectra as seen in Figure 1, with $10^{-4.4}$ of the stellar luminosity emerging from those chromospheric cooling lines, $\log R_{\mathrm{HK}}^{\prime}=-4.4$ (Wright et al. 2004). This strong emission implies a young age of $0.5-1.0 \mathrm{Gyr}$, similar to Hyades stars. We expect this star to exhibit photospheric velocity jitter of $9 \pm 3 \mathrm{~m} \mathrm{~s}^{-1}$ (Wright et al. 2005) based on other similar, young $\mathrm{K} 0$ stars on our program. This large jitter is probably caused by magnetically influenced turbulent flows and spots on the rotating stellar surface (Saar et al. 1998; Paulson et al. 2004). HD 128311 has properties and jitter similar to those of HD 192263 for which the planet (Vogt et al. 2000; Santos et al. 2000b, 2003) now seems well supported. For such young, magnetically active stars, our estimate of jitter is uncertain by at least $30 \%$. The mag- netic cycles and turbulence of such young stars prevent a more secure prediction of jitter. We estimate the stellar mass of HD 128311 to be $M=0.84 M_{\odot}$ from the star's position on the H-R diagram and its metallicity, employing the stellar evolution models of Girardi et al. (2002) and Fischer \& Valenti (2005).

Table 3 lists the 76 observations for HD 128311, giving the time of each observation, the measured radial velocity, and the internal error stemming from the weighted uncertainty in the mean of the 400 spectral chunks of length $2 \AA$ that were Doppler analyzed. Figure 3 (bottom panel) shows the measured relative velocities for HD 128311 as filled circles with associated uncertainties. A model consisting of a single Keplerian orbit (not shown) yields residuals with rms $=33 \mathrm{~m} \mathrm{~s}^{-1}$, so much larger than errors and jitter that it is ruled out. A model consisting of the sum of two independent Keplerian orbits yields a lower rms of $18 \mathrm{~m} \mathrm{~s}^{-1}$ and $\left(\chi_{\nu}^{2}\right)^{1 / 2}=1.86$. We compute $\left(\chi_{\nu}^{2}\right)^{1 / 2}$ by adopting the expected velocity "noise" to be the quadrature sum of internal errors $\left(\sim 3 \mathrm{~m} \mathrm{~s}^{-1}\right)$ and predicted photospheric jitter $\left(\sim 9 \mathrm{~m} \mathrm{~s}^{-1}\right)$. A successful model should have $\left(\chi_{\nu}^{2}\right)^{1 / 2}$ near unity, but our predictions of jitter are accurate only to within $50 \%$, preventing any strong interpretation of $\left(\chi_{\nu}^{2}\right)^{1 / 2}$.

The top two panels of Figure 3 show the velocities and orbital fits for each planet individually, by subtracting the variations due to the other planet. The best double-Keplerian model is shown in the bottom panel of Figure 3. The two-planet fit gives orbital periods of $P=458.6$ and 928.3 days, velocity semiamplitudes of $K=67$ and $76 \mathrm{~m} \mathrm{~s}^{-1}$, and eccentricities of $e=$ 0.25 and 0.17 , respectively (see Table 2 ). The implied minimum masses of the companions are $M \sin i=2.18 M_{\mathrm{J}}$ and $3.21 M_{\mathrm{J}}$. The two orbital periods have a ratio of 2.024 , raising the strong suggestion of gravitational interactions and a mean motion dynamical resonance. Preliminary three-body simulations in $\S 5$ indeed suggest a 2:1 mean motion resonance, with the timescale for significant orbital changes being decades. We are carrying out further simulations, to be reported in G. Laughlin et al. (2005, in preparation).

A periodogram of the velocity residuals to the two-planet fit exhibits a strong peak at a period of 5.60 days, with a false alarm 
TABLE 3

Radial Velocities for HD 128311

\begin{tabular}{|c|c|c|}
\hline $\begin{array}{c}\text { JD } \\
(-2,440,000)\end{array}$ & $\begin{array}{c}\mathrm{RV} \\
\left(\mathrm{m} \mathrm{s}^{-1}\right)\end{array}$ & $\begin{array}{c}\text { Uncertainty } \\
\left(\mathrm{m} \mathrm{s}^{-1}\right)\end{array}$ \\
\hline 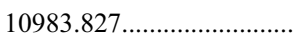 & -27.50 & 2.8 \\
\hline $11200.138 \ldots \ldots \ldots \ldots \ldots \ldots \ldots$ & -33.44 & 3.7 \\
\hline $11311.927 \ldots \ldots \ldots \ldots \ldots \ldots \ldots$ & 59.71 & 13.5 \\
\hline 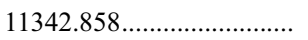 & 48.02 & 4.3 \\
\hline $11370.829 \ldots \ldots \ldots \ldots \ldots \ldots \ldots$ & 91.67 & 3.7 \\
\hline 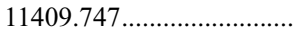 & 111.56 & 3.2 \\
\hline $11410.749 \ldots \ldots \ldots \ldots \ldots \ldots \ldots$ & 103.97 & 4.1 \\
\hline $11552.165 \ldots \ldots \ldots \ldots \ldots \ldots \ldots \ldots$. & 56.62 & 3.7 \\
\hline $11581.170 \ldots \ldots \ldots \ldots \ldots \ldots \ldots \ldots$ & 1.19 & 3.2 \\
\hline $11680.025 \ldots \ldots \ldots \ldots \ldots \ldots .$. & -75.54 & 4.4 \\
\hline $11974.161 \ldots \ldots \ldots \ldots \ldots \ldots \ldots . .$. & 48.65 & 3.6 \\
\hline $11982.153 \ldots \ldots \ldots \ldots \ldots \ldots \ldots \ldots$ & 18.48 & 3.0 \\
\hline $12003.023 \ldots \ldots \ldots \ldots \ldots \ldots \ldots . .$. & -2.08 & 3.9 \\
\hline 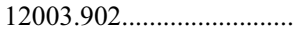 & 14.26 & 3.9 \\
\hline 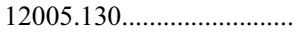 & 12.95 & 3.0 \\
\hline $12061.878 \ldots \ldots \ldots \ldots \ldots \ldots$ & -55.37 & 3.1 \\
\hline $12062.867 \ldots \ldots \ldots \ldots \ldots \ldots \ldots . .$. & -26.31 & 3.4 \\
\hline $12096.776 \ldots \ldots \ldots \ldots \ldots \ldots . .$. & -42.70 & 3.5 \\
\hline 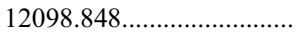 & -44.02 & 3.0 \\
\hline $12128.766 \ldots \ldots \ldots \ldots \ldots \ldots . .$. & -13.61 & 3.8 \\
\hline $12162.724 \ldots$ & 5.64 & 3.4 \\
\hline $12308.173 \ldots \ldots \ldots \ldots \ldots \ldots \ldots \ldots$ & 133.24 & 3.4 \\
\hline $12333.160 \ldots \ldots \ldots \ldots \ldots$ & 103.14 & 3.4 \\
\hline $12335.118 \ldots \ldots \ldots \ldots \ldots \ldots$ & 96.98 & 3.4 \\
\hline $12362.994 \ldots \ldots \ldots \ldots$ & 133.22 & 3.3 \\
\hline 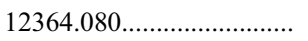 & 131.45 & 3.1 \\
\hline 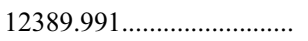 & 87.16 & 3.4 \\
\hline $12390.957 \ldots \ldots \ldots \ldots \ldots \ldots$ & 91.67 & 3.5 \\
\hline $12445.826 \ldots \ldots \ldots \ldots \ldots \ldots . .$. & 70.15 & 3.2 \\
\hline $12486.827 \ldots \ldots \ldots \ldots \ldots \ldots . .$. & 9.55 & 2.9 \\
\hline 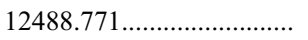 & 27.15 & 3.4 \\
\hline $12515.731 \ldots \ldots \ldots \ldots \ldots \ldots .$. & -42.47 & 3.3 \\
\hline $12653.176 \ldots \ldots \ldots \ldots \ldots \ldots \ldots . .$. & -89.74 & 3.3 \\
\hline $12654.160 \ldots \ldots \ldots \ldots \ldots \ldots \ldots . .$. & -103.97 & 3.9 \\
\hline 12681.161....................... & -101.86 & 3.4 \\
\hline 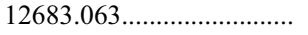 & -75.81 & 3.2 \\
\hline $12712.005 \ldots \ldots \ldots \ldots \ldots \ldots \ldots \ldots$ & -43.13 & 3.1 \\
\hline 12712.974 & -56.45 & 3.9 \\
\hline $12776.961 \ldots \ldots \ldots \ldots \ldots \ldots$ & -53.89 & 3.2 \\
\hline $12777.884 \ldots \ldots \ldots \ldots \ldots \ldots \ldots$ & -35.71 & 3.0 \\
\hline $12803.898 \ldots \ldots \ldots \ldots \ldots \ldots . .$. & -41.99 & 3.0 \\
\hline $12804.958 \ldots \ldots \ldots \ldots \ldots \ldots . .$. & -45.16 & 2.6 \\
\hline 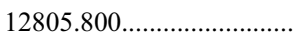 & -30.43 & 3.4 \\
\hline 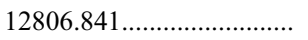 & -25.00 & 2.7 \\
\hline 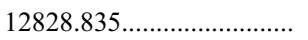 & -28.04 & 2.7 \\
\hline 12832.739 & -22.05 & 2.9 \\
\hline $12833.783 \ldots \ldots \ldots \ldots \ldots$ & -14.23 & 3.3 \\
\hline $12834.868 \ldots \ldots \ldots \ldots \ldots \ldots . .$. & 13.79 & 2.9 \\
\hline $12848.795 \ldots \ldots \ldots \ldots \ldots \ldots . .$. & -7.46 & 2.7 \\
\hline 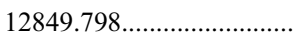 & -21.94 & 2.7 \\
\hline 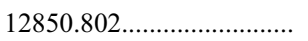 & -17.30 & 3.0 \\
\hline 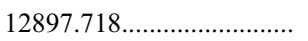 & 25.41 & 3.4 \\
\hline $13015.152 \ldots \ldots \ldots \ldots \ldots \ldots \ldots . .$. & 5.58 & 2.8 \\
\hline $13016.170 \ldots \ldots \ldots \ldots \ldots \ldots . .$. & -45.05 & 2.8 \\
\hline $13017.157 \ldots \ldots \ldots \ldots \ldots \ldots \ldots . .$. & -86.32 & 2.9 \\
\hline 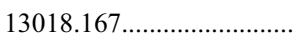 & -55.74 & 3.1 \\
\hline $13046.174 \ldots \ldots \ldots \ldots \ldots \ldots \ldots . .$. & -44.54 & 3.0 \\
\hline 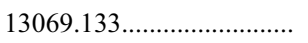 & -13.16 & 2.9 \\
\hline 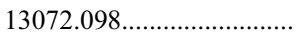 & 1.58 & 3.0 \\
\hline 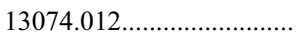 & 0.00 & 3.1 \\
\hline $13077.144 \ldots \ldots \ldots \ldots \ldots \ldots \ldots \ldots . .$. & -17.01 & 3.6 \\
\hline 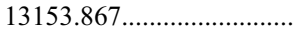 & 58.35 & 2.8 \\
\hline $13179.850 \ldots \ldots \ldots \ldots \ldots \ldots \ldots$ & 65.59 & 2.9 \\
\hline $13180.819 \ldots \ldots \ldots \ldots \ldots \ldots$ & 84.71 & 2.6 \\
\hline
\end{tabular}

TABLE 3-Continued

\begin{tabular}{|c|c|c|}
\hline $\begin{array}{c}\text { JD } \\
(-2,440,000)\end{array}$ & $\begin{array}{c}\mathrm{RV} \\
\left(\mathrm{m} \mathrm{s}^{-1}\right)\end{array}$ & $\begin{array}{c}\text { Uncertainty } \\
\left(\mathrm{m} \mathrm{s}^{-1}\right)\end{array}$ \\
\hline $13181.814 \ldots \ldots \ldots \ldots \ldots \ldots \ldots$ & 95.71 & 2.7 \\
\hline $13195.801 \ldots \ldots \ldots \ldots \ldots \ldots \ldots \ldots . .$. & 72.87 & 2.7 \\
\hline 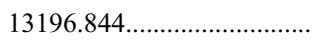 & 97.85 & 2.8 \\
\hline $13238.759 \ldots \ldots \ldots \ldots \ldots \ldots$ & 101.55 & 2.8 \\
\hline $13239.750 \ldots$ & 96.94 & 2.8 \\
\hline $13240.793 \ldots \ldots \ldots \ldots \ldots \ldots$ & 100.17 & 3.1 \\
\hline $13369.169 \ldots \ldots \ldots \ldots \ldots \ldots \ldots$ & 83.11 & 2.7 \\
\hline 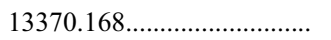 & 92.97 & 3.0 \\
\hline $13425.109 \ldots \ldots \ldots \ldots \ldots \ldots \ldots$ & -13.28 & 2.9 \\
\hline $13479.986 \ldots \ldots \ldots \ldots \ldots \ldots \ldots$ & -66.31 & 2.7 \\
\hline $13480.828 \ldots \ldots \ldots \ldots \ldots \ldots$ & -48.76 & 2.8 \\
\hline $13483.858 \ldots \ldots \ldots \ldots \ldots$. & -86.30 & 2.7 \\
\hline
\end{tabular}

probability of less than $1 \%$. We suspect that this periodicity is not caused by a third planet but instead is photospheric. Optical photometry by Strassmeier et al. (2000) revealed a period of 11.5 days with an amplitude of 0.035 mag. We independently find a photometric period that is nearly twice 5.6 days, as described in $\S 4$, suggesting a rotation period of 11.5 days. Such photometric variations in young stars are associated with velocity jitter of $10-20 \mathrm{~m} \mathrm{~s}^{-1}$ (Paulson et al. 2004). From the emission at $\mathrm{Ca}$ II $\mathrm{H}$ and $\mathrm{K}$, we predict the rotation period to be $P_{\text {rot }}=12$ days, in agreement with the photometric period. Thus, we suspect that the observed velocity period of 5.6 days is caused by spots on opposite hemispheres of the star rotating across the visible stellar disk, alternately blocking the approaching and receding limbs of the star and causing a net Doppler shift.

\section{2. $H D 108874$}

Our previous velocities for HD 108874 (HIP 61028) obtained from 1999 to 2002 revealed a planet with a period of 398 days, eccentricity of 0.17 , semiamplitude $K=48 \mathrm{~m} \mathrm{~s}^{-1}$, and a minimum mass of $M \sin i=1.7 M_{\mathrm{J}}$ (Butler et al. 2003). The best-fit Keplerian model demanded inclusion of a linear trend in velocity with a slope of $+7 \mathrm{~m} \mathrm{~s}^{-1} \mathrm{yr}^{-1}$, indicating an outer companion of unknown mass and orbital period. That upward trend reached a maximum velocity in 2003 and has declined since, providing new physical constraints on this second orbiting companion, rendering it likely planetary.

We have revised our estimates of the stellar properties for HD 108874. Remaining unchanged are estimates of spectral type G5 V, parallax of 14.6 mas (ESA 1997), and $M_{V}=4.58$, placing the star on the main sequence (see Table 1). However, new LTE spectral synthesis of our HIRES spectra shows that $[\mathrm{Fe} / \mathrm{H}]=+0.18$, and measurements of $\mathrm{Ca}$ II $\mathrm{H}$ and $\mathrm{K}$ give $\log R_{\mathrm{HK}}^{\prime}=-5.07$, which implies an expected velocity jitter of $3.9 \mathrm{~m} \mathrm{~s}^{-1}$ (Valenti \& Fischer 2005; Wright et al. 2004, 2005). From its $B-V$ of $0.71, M_{V}$, metallicity, and the models of Girardi et al. (2002), our best estimate of the stellar mass is $M=0.99 \pm 0.1 M_{\odot}$.

Table 4 gives the time, measured radial velocity, and internal error of each of the 49 HIRES observations. Figure 4 (bottom panel) shows the measured relative velocities for HD 108874 and the associated best-fit double-Keplerian model. The model is very good, with $\left(\chi_{\nu}^{2}\right)^{1 / 2}=0.79$ and $\mathrm{rms}$ of the residuals of $3.7 \mathrm{~m} \mathrm{~s}^{-1}$. The previously detected inner planet has a revised orbital period of $P_{b}=395.4$ days, nearly the same as before, 

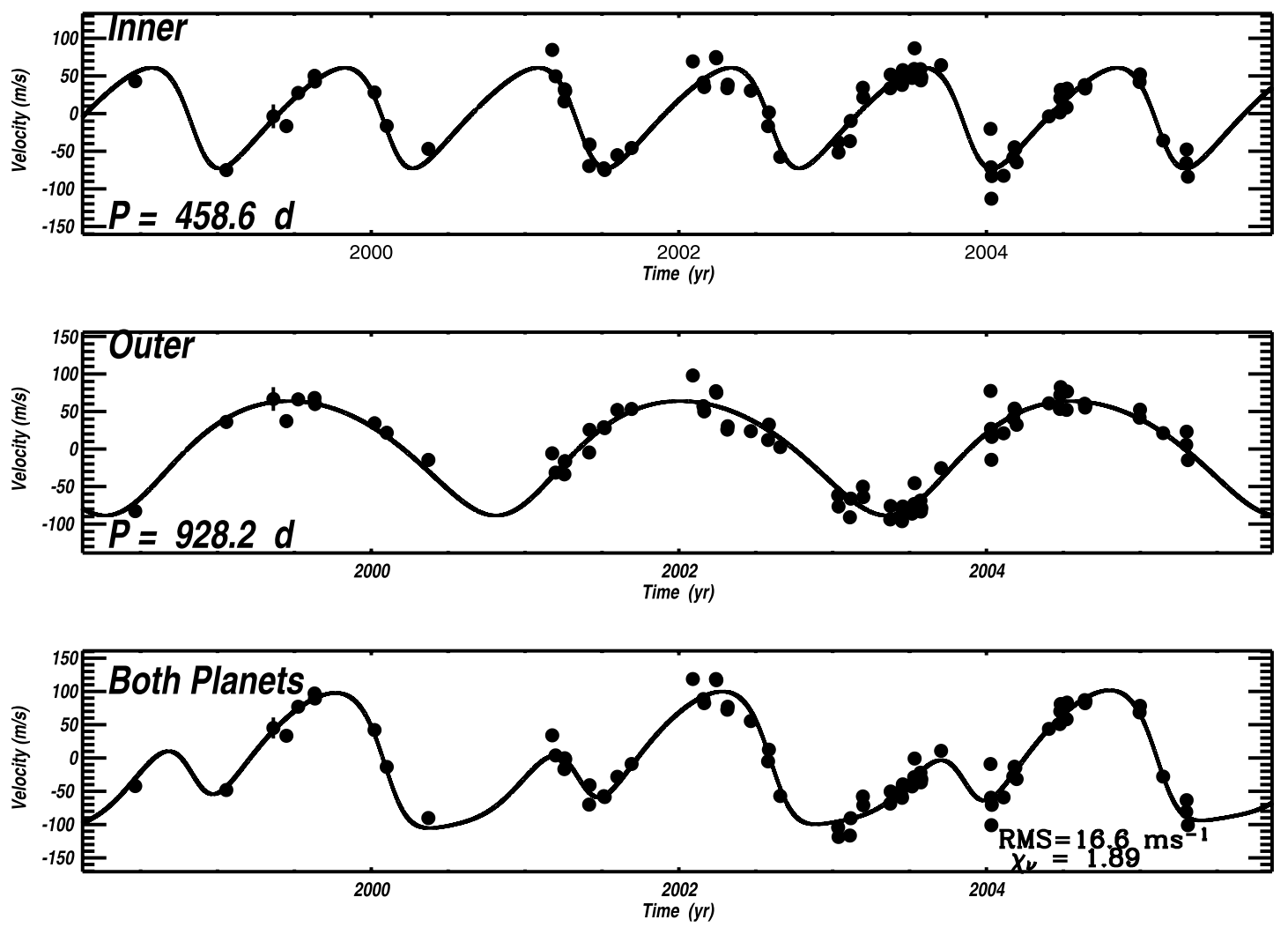

Fig. 3.-Measured velocities ( filled circles) vs. time for HD 128311. Top: Velocity vs. time ( filled circles) for the inner planet with the effects of the outer planet subtracted. The associated best-fit orbit is shown (solid line). Middle: Velocity vs. time (filled circles) for the outer planet with the effects of the inner planet subtracted. Bottom: Original velocities ( filled circles) and the best-fit two-Keplerian orbit consisting of periods $P=458.6$ and 928.3 days and minimum masses $M \sin i=2.18 M_{\mathrm{J}}$ and $3.21 M_{\mathrm{J}}$. Errors bars include the quadrature sum of internal uncertainties $\left(\sim 3 \mathrm{~m} \mathrm{~s}^{-1}\right)$ and expected jitter of $8.9 \mathrm{~m} \mathrm{~s} \mathrm{~s}^{-1}$. No planet-planet interactions are included in the fit above, but dynamical simulations suggest a $2: 1$ resonance.

but the eccentricity is now $e_{b}=0.07$ and the semiamplitude is $K_{b}=38.0 \mathrm{~m} \mathrm{~s}^{-1}$, implying a minimum mass of $M \sin i=$ $1.36 M_{\mathrm{J}}$, somewhat lower than we derived previously (Butler et al. 2003).

To view the outer planet explicitly, we have subtracted the effects of the inner planet, as shown in the middle panel of Figure 4. Apparently, this outer planet has just completed one full orbit. The two-Keplerian fit yields $P_{c}=1605.8$ days $=$ $4.4 \mathrm{yr}, e_{c}=0.25, K_{c}=17.3 \mathrm{~m} \mathrm{~s}^{-1}$, and minimum mass $M_{c} \sin i_{c}=1.02 M_{\mathrm{J}}$.

Several additional years of measurements will be required to constrain further the orbit of the outer planet. The semimajor axis of this outer planet is $2.7 \mathrm{AU}$, implying an angular separation from the star at apastron of up to $0^{\prime \prime} 049$. This angular separation is probably too small to permit direct detection of the planet with upcoming adaptive optics or space-borne coronagraphic cameras (e.g., Trauger et al. 2003), but it could be detected astrometrically with the Space Interferometry Mission (Shao 2003) and by the Terrestrial Planet Finder and Darwin.

\subsection{HD 217107}

HD 217107 (HIP 113421) was previously reported to have a planet with orbital period 7.1 days and minimum mass of $1.4 M_{\mathrm{J}}$ (Fischer et al. 1999) based on velocities from both Lick and Keck observatories, and a superimposed linear trend in the radial velocities was described in Fischer et al. (2001). The bestfit eccentricity to the inner planet was $e=0.13$, larger than the eccentricity of the majority of hot Jupiters with periods less than 11 days. Indeed, exoplanets with $P<11$ days have much smaller eccentricities than the others, suggesting that tidal circularization is acting. As short-period planets are tidally circularized, the nonzero eccentricity and the linear trend of HD $217107 \mathrm{~b}$ suggest the presence of a more distant orbiting object that pumps the eccentricity. During the past $2 \mathrm{yr}$, that linear velocity trend now exhibits significant curvature, providing constraints on, and a preliminary orbit for, this second companion.

HD 217107 has a spectral type G8 IV, a parallax of 50.7 mas (ESA 1997), and $M_{V}=4.71$, placing it a few tenths of a magnitude above the main sequence (see Table 1). Analysis of our HIRES spectra shows $[\mathrm{Fe} / \mathrm{H}]=+0.32, \log R_{\mathrm{HK}}^{\prime}=-5.08$, and an expected jitter of $3.4 \mathrm{~m} \mathrm{~s}^{-1}$ (Valenti \& Fischer 2005; Wright et al. 2004, 2005). From its $B-V$ of $0.72, M_{V}$, metallicity, and the models of Girardi et al. (2002), we find the stellar mass to be $M=1.053 \pm 0.1 M_{\odot}$.

The 63 velocity measurements for HD 217107 from the Keck telescope are listed in Table 5, giving the time, velocity, and internal error of each observation. Figure 5 shows those measured relative velocities and the associated best-fit double-Keplerian model. The model yields residuals having $\mathrm{rms}=5.1 \mathrm{~m} \mathrm{~s}^{-1}$ and $\left(\chi_{\nu}^{2}\right)^{1 / 2}=1.1$, representing a satisfactory fit. The orbit and mass of the inner planet are changed very little from the best-fit orbit of Fischer et al. (2001). The current best-fit parameters are $P_{b}=7.1269$ days, $e_{b}=0.13 \pm 0.02, K_{b}=140.7 \mathrm{~m} \mathrm{~s}^{-1}$, implying a minimum mass of $M_{b} \sin i_{b}=1.37 M_{\mathrm{J}}$.

Subtracting the signal caused by the inner planet from both the measured velocities and the model reveals the effects of the outer companion. Figure 5 shows that the residual velocities rose steeply during three seasons, from 1998.7 to 2000.5 , but have leveled off during the past three seasons, from 2002.5 to 2005.0. Thus, the orbital period for component " $\mathrm{c}$ " is $>6 \mathrm{yr}$ but 
TABLE 4

Radial VeLocities FOR HD 108874

\begin{tabular}{|c|c|c|}
\hline $\begin{array}{c}\text { JD } \\
(-2,440,000)\end{array}$ & $\begin{array}{c}\mathrm{RV} \\
\left(\mathrm{m} \mathrm{s}^{-1}\right)\end{array}$ & $\begin{array}{c}\text { Uncertainty } \\
\left(\mathrm{m} \mathrm{s}^{-1}\right)\end{array}$ \\
\hline $11340.806 \ldots \ldots \ldots \ldots \ldots \ldots$ & 26.94 & 5.0 \\
\hline 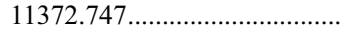 & 43.48 & 2.8 \\
\hline $11373.751 \ldots \ldots \ldots \ldots \ldots \ldots \ldots \ldots$ & 47.32 & 3.5 \\
\hline 11581.070 & -0.22 & 4.0 \\
\hline 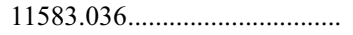 & -12.06 & 3.6 \\
\hline $11585.106 \ldots \ldots \ldots \ldots \ldots \ldots \ldots \ldots \ldots \ldots$ & -16.38 & 2.7 \\
\hline $11585.995 \ldots \ldots \ldots \ldots \ldots \ldots \ldots \ldots . .$. & -15.84 & 3.2 \\
\hline 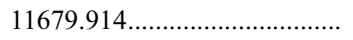 & -24.15 & 3.1 \\
\hline 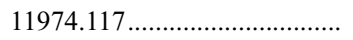 & -14.40 & 2.6 \\
\hline $11982.046 \ldots \ldots \ldots \ldots \ldots \ldots \ldots \ldots$ & -25.17 & 3.5 \\
\hline$\ldots$ & -25.65 & 3.6 \\
\hline $12009.039 \ldots$. & -33.35 & 4.1 \\
\hline 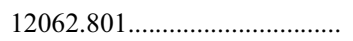 & -35.74 & 3.2 \\
\hline $12097.815 \ldots$ & -13.01 & 2.9 \\
\hline $12307.991 \ldots$ & 23.93 & 4.0 \\
\hline 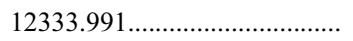 & 9.57 & 4.0 \\
\hline 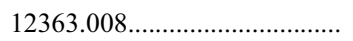 & 3.50 & 3.2 \\
\hline $12364.029 \ldots \ldots \ldots \ldots \ldots \ldots \ldots \ldots . .$. & 4.35 & 2.6 \\
\hline $12389.979 \ldots \ldots \ldots \ldots \ldots \ldots \ldots$ & -17.70 & 4.1 \\
\hline 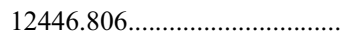 & -13.51 & 3.0 \\
\hline 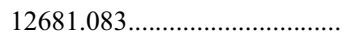 & 56.65 & 3.6 \\
\hline $12711.951 \ldots \ldots \ldots \ldots \ldots \ldots \ldots$ & 46.75 & 3.4 \\
\hline 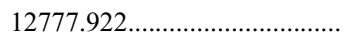 & 11.70 & 3.4 \\
\hline 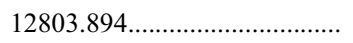 & 2.66 & 3.4 \\
\hline 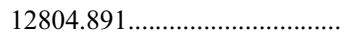 & 10.15 & 3.3 \\
\hline $12805.809 \ldots \ldots \ldots \ldots \ldots \ldots \ldots \ldots$ & 4.52 & 3.5 \\
\hline 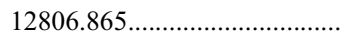 & 6.00 & 3.2 \\
\hline 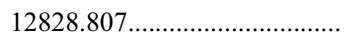 & -0.42 & 2.8 \\
\hline $12832.752 \ldots \ldots \ldots \ldots \ldots \ldots \ldots . .$. & -0.55 & 3.5 \\
\hline $12833.778 \ldots \ldots \ldots \ldots \ldots \ldots \ldots . .$. & 0.00 & 3.0 \\
\hline $12848.744 \ldots \ldots \ldots \ldots \ldots \ldots \ldots \ldots$ & -2.80 & 3.6 \\
\hline $12850.780 \ldots \ldots \ldots \ldots \ldots \ldots \ldots \ldots$ & -3.93 & 3.7 \\
\hline 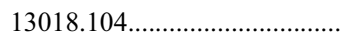 & 57.18 & 3.4 \\
\hline 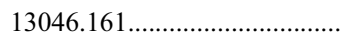 & 50.20 & 3.6 \\
\hline 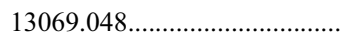 & 38.23 & 4.9 \\
\hline 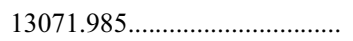 & 43.29 & 3.5 \\
\hline 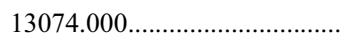 & 39.50 & 3.3 \\
\hline $13077.096 \ldots \ldots \ldots \ldots \ldots \ldots \ldots \ldots$ & 38.96 & 3.5 \\
\hline 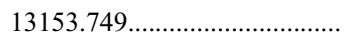 & -4.62 & 3.1 \\
\hline $13179.767 \ldots \ldots$ & -18.37 & 3.4 \\
\hline 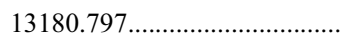 & -23.98 & 3.4 \\
\hline 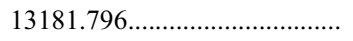 & -18.98 & 3.0 \\
\hline $13189.836 \ldots \ldots \ldots \ldots \ldots \ldots \ldots \ldots \ldots$ & -21.02 & 3.1 \\
\hline $13195.781 \ldots \ldots \ldots \ldots \ldots \ldots \ldots \ldots$ & -27.73 & 2.7 \\
\hline 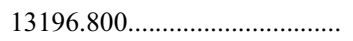 & -24.75 & 2.9 \\
\hline 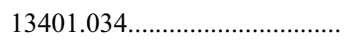 & 42.95 & 3.0 \\
\hline 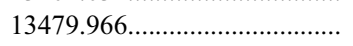 & 26.63 & 3.1 \\
\hline $13480.832 \ldots \ldots \ldots \ldots \ldots \ldots \ldots \ldots \ldots$ & 21.16 & 3.0 \\
\hline $13483.807 \ldots \ldots$ & 19.62 & 3.4 \\
\hline
\end{tabular}

probably less than 4 times that duration, unless the eccentricity is extreme.

The orbit for component "c" remains quite uncertain and only modestly constrained. However, even such poor constraints may help direct the timing and precision of future Doppler work, and they may help interpret data from other detection techniques such as astrometry and direct imaging (Brown 2004). Motivated by such value, we carried out a two-planet fit to the Keck velocities. The best-fit orbital parameters for the outer companion are $P_{c}=3150$ days $=8.6 \mathrm{yr}, e_{c}=0.55, K_{c}=34.4 \mathrm{~m} \mathrm{~s}^{-1}$, implying a minimum mass of $M_{c} \sin i_{c}=2.1 M_{\mathrm{J}}$. The residuals to the two-planet fit have rms $=5.07 \mathrm{~m} \mathrm{~s}^{-1}$ and $\left(\chi_{\nu}^{2}\right)^{1 / 2}=1.1$. While the fit is good, equally good fits can be obtained with considerably longer periods, up to $P=20 \mathrm{yr}$ and beyond. The best-fit period of $8.4 \mathrm{yr}$ is longer than the duration of Keck observations, $6.5 \mathrm{yr}$, leaving the possibility that the period is considerably longer. If so, the planet mass would be somewhat larger than the $2.1 M_{\mathrm{J}}$ found in this model. As we observe significant curvature in the velocities from the outer companion, it is unlikely that the period is more than 4 times longer than the observations: $P<26$ yr. The best-fit semimajor axis for planet "c" (from Kepler's third law) is $a_{c}=4.3 \mathrm{AU}$, also a lower limit. All orbital parameters are listed in Table 2.

We have also continued to obtain velocities for HD 217107 from Lick Observatory, contiguous with the velocity set described by Fischer et al. (2001). The Lick data and the best-fit two-planet orbit are shown in Figure 6, and Table 2 lists the resulting orbital parameters that are derived separately from the Lick velocities alone. The best-fit orbital parameters for the inner planet are identical to those from Keck, providing secure confirmation. The outer planet has a best-fit period of 2466 days $=6.8 \mathrm{yr}$, eccentricity of 0.53 , and $M \sin i$ of $3.31 M_{\mathrm{J}}$. The rms to the residuals is $10.0 \mathrm{~m} \mathrm{~s}^{-1}$ and the reduced $\left(\chi_{\nu}^{2}\right)^{1 / 2}$ is 1.70 , both indicating that the residuals are somewhat larger than the expected errors and jitter. The orbital period and eccentricity are within $20 \%$ of the values found from the Keck velocities. However, the velocity semiamplitude of $57 \mathrm{~m} \mathrm{~s}^{-1}$ is much higher than that from Keck $\left(34.4 \mathrm{~m} \mathrm{~s}^{-1}\right)$, an indication of the magnitude of the uncertainty in this outer companion.

To assess the uncertainty in the orbital parameters of the outer companion, we have sampled the range of parameter values from $30 \%$ to a factor of 3 of all orbital parameters for the twoKeplerian model. Orbital parameters that yield $\left(\chi_{\nu}^{2}\right)^{1 / 2}$ within unity of its best-fit value are deemed plausible (albeit unlikely at the extremes). We find that the orbital period could be up to 3 times its best-fit value of $8.4 \mathrm{yr}$ and still yield $\left(\chi_{\nu}^{2}\right)^{1 / 2}<2.5$. Similarly, the value of $K$ could be roughly twice its best-fit values of $34-57 \mathrm{~m} \mathrm{~s}^{-1}$. Thus, the orbital semimajor axis could be between 3.6 and 7.4 AU and the minimum mass could be between $1.8 M_{\mathrm{J}}$ and $6 M_{\mathrm{J}}$. These uncertainties are large but suggest that the companion is of planetary mass (less than $13 M_{\mathrm{J}}$ ) rather than stellar $\left(M>80 M_{\mathrm{J}}\right)$ or brown dwarf. An image of HD 217107 taken with the Keck telescope and adaptive optics shows no evidence of any stellar companion having separation greater than 0.1 (M. Liu 2005, private communication). There is little hope of significant improvement in the mass and orbit measurements until another few years of Doppler data are obtained. In summary, the reality of this outer companion is not in doubt and the curvature strongly suggests an orbit of 3-8 AU and a planetary mass, making this another planet orbiting at distances comparable to the Jovian planets in our solar system.

Based on these best-fit orbits for HD 217107 from Keck and Lick, the outer planet at apastron reaches an angular separation of at least 0.31 and possibly larger if the period is longer than $7 \mathrm{yr}$. Thus, this planet offers an opportunity for direct detection with extreme adaptive optics from ground-based telescopes, space-borne coronagraphic cameras, and interferometers. Velocities obtained during the next few years will help constrain the parameters of this outer planet (Brown 2004).

\subsection{HD 50499}

HD 50499 (HIP 32970) has a spectral type G1 V, a parallax of 21.2 mas (ESA 1997), and $M_{V}=3.85$, placing it $0.6 \mathrm{mag}$ above the main sequence (Table 1). Analysis of our HIRES spectra shows that $[\mathrm{Fe} / \mathrm{H}]=+0.30, \log R_{\mathrm{HK}}^{\prime}=-5.02$, and the jitter is predicted to be $2.8 \mathrm{~m} \mathrm{~s}^{-1}$ (Valenti \& Fischer 2005; Wright et al. 

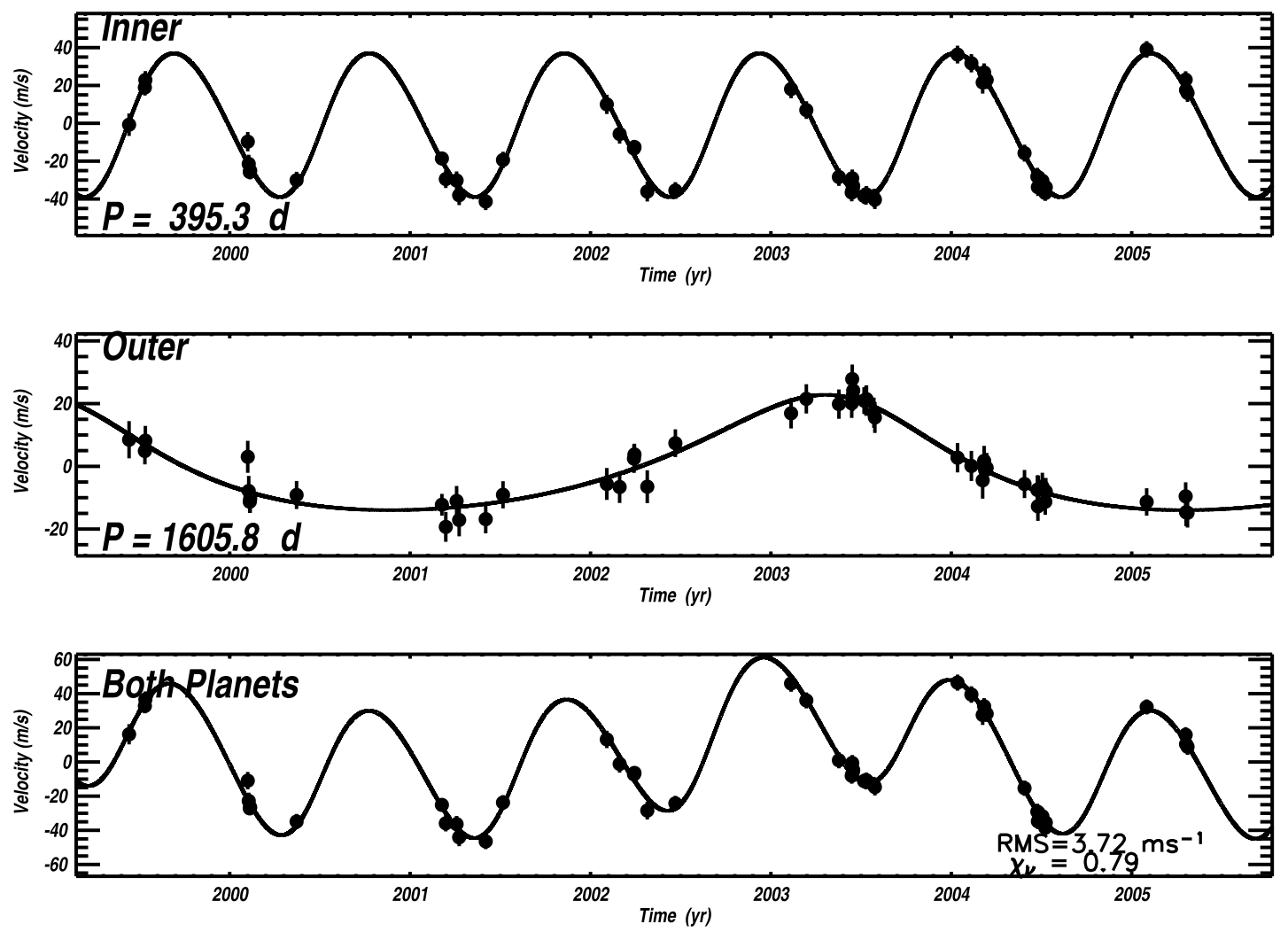

FIG. 4.- Measured velocities ( filled circles) vs. time for HD 108874. Top: Inner planet alone with effects of the outer planet removed. Middle: Outer planet alone. The associated orbit for each planet is shown by the solid line. Bottom: Original velocities ( filled circles) and the best-fit two-Keplerian model consisting of periods $P=395.4$ and 1605.8 days and minimum masses $M \sin i=1.36 M_{\mathrm{J}}$ and $1.02 M_{\mathrm{J}}$. Error bars include the quadrature sum of internal uncertainties and jitter, and the residuals have $\mathrm{rms}=3.7 \mathrm{~m} \mathrm{~s}^{-1}$ and $\left(\chi_{\nu}^{2}\right)^{1 / 2}=0.79$.

2004, 2005). Henry et al. (1996) found $\log R_{\mathrm{HK}}^{\prime}=-5.06$, similar to that here. From its $B-V$ of $0.575, M_{V}$, metallicity, and the models of Girardi et al. (2002), the stellar mass is $M=$ $1.24 \pm 0.1 M_{\odot}$. This star is older than $6 \mathrm{Gyr}$, by virtue of its low $R_{\mathrm{HK}}^{\prime}$ (Noyes et al. 1984; Wright 2004), and metal-rich, both of which explain its position well above the main sequence.

We have obtained 35 velocities for HD 50499 from 1996 December through 2005 April. Table 6 gives the time, velocity, and internal uncertainty of each measurement, with the median internal error being $3.7 \mathrm{~m} \mathrm{~s}^{-1}$. Visual inspection of the velocities reveals clear temporal coherence, including a local minimum in 1998, a subsequent maximum in 2000 , and a current downward trend with velocities extending below those at the 1998 minimum. Figure 7 shows the measured relative velocities for HD 50499.

By inspection, the simplest model contains an orbiting, Keplerian companion with an additional downward linear velocity trend. The best-fit such model is shown in Figure 7 by a dashed line. The resulting orbital parameters are $P=2483$ days $=$ $6.8 \mathrm{yr}, e=0.23, K=22.9 \mathrm{~m} \mathrm{~s}^{-1}$, implying a minimum mass $M \sin i=1.71 M_{\mathrm{J}}$. The linear trend is $-4.8 \mathrm{~m} \mathrm{~s}^{-1} \mathrm{yr}^{-1}$, indicating the presence of an additional companion. The residuals to the fit have rms $=4.78 \mathrm{~m} \mathrm{~s}^{-1}$ and $\left(\chi_{\nu}^{2}\right)^{1 / 2}=1.07$, both indicating that the residuals are consistent with the velocity uncertainties and jitter. This Keplerian fit reveals a planet, not previously known, with a semimajor axis of $a=3.86 \mathrm{AU}$.

The trend of $-4.8 \mathrm{~m} \mathrm{~s}^{-1} \mathrm{yr}^{-1}$ lasting the duration of the observations, $8.4 \mathrm{yr}$, indicates the existence of a second companion having semimajor axis of at least $4 \mathrm{AU}$ and minimum mass of at least $2 M_{\mathrm{J}}$. A brown dwarf or stellar companion located within
100 AU could explain the linear trend. However, the Hipparcos catalog shows that the fits to its astrometric model of parallax and proper motion carried residuals of $\sim 0.7$ mas during the $4 \mathrm{yr}$ mission lifetime. These small residuals are consistent with known astrometric errors (ESA 1997). Thus, Hipparcos imposes an upper limit to the mass of any companion having a period between 5 and 15 yr of roughly $50 M_{\mathrm{J}}$, i.e., ruling out stellar companions within $7 \mathrm{AU}$. Indeed, brown dwarf and stellar companions with periods of 5-15 yr can also be ruled out due to the stable orbit of the inner planet with its period of 6.8 yr. However, a brown dwarf or low-mass M dwarf orbiting beyond $10 \mathrm{AU}$ from the star would be absorbed into the proper motion of the model of Hipparcos data and might leave the inner planet in a stable orbit. Thus, the linear trend might be due to a second planet, a brown dwarf, or a low-mass star beyond $10 \mathrm{AU}$. This outer companion of unknown mass and orbit warrants follow-up by other techniques, such as imaging with advanced adaptive optics, interferometric sensing with VLT or Keck, astrometric measurements with precision under 1 mas, and space-based imaging.

We tried two-planet models consisting of an inner planet similar to that in the single-planet solution and an outer planet with a period longer than $15 \mathrm{yr}$ (explaining the linear trend). The best fit is achieved with two planets having $P=9.9$ and $37 \mathrm{yr}$ and minimum masses of $3.4 M_{\mathrm{J}}$ and $2.1 M_{\mathrm{J}}$, respectively. That is, the outer companion may well be planetary in nature. However, the two-planet solution does not reduce the value of $\left(\chi_{\nu}^{2}\right)^{1 / 2}$, suggesting that this model is not superior to a model consisting of one planet and a linear velocity trend. More observations are needed to constrain the outer companion in this system. 
TABLE 5

RADIAL Velocities FOR HD 217107

\begin{tabular}{|c|c|c|}
\hline $\begin{array}{c}\text { JD } \\
(-2,440,000)\end{array}$ & $\begin{array}{c}\mathrm{RV} \\
\left(\mathrm{m} \mathrm{s}^{-1}\right)\end{array}$ & $\begin{array}{c}\text { Uncertainty } \\
\left(\mathrm{m} \mathrm{s}^{-1}\right)\end{array}$ \\
\hline 11068.860 & -68.74 & 2.2 \\
\hline 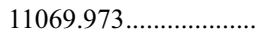 & -163.05 & 2.8 \\
\hline 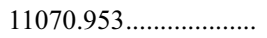 & -168.73 & 1.8 \\
\hline $11071.869 \ldots$ & -118.40 & 2.7 \\
\hline 11072.929. & -10.12 & 3.2 \\
\hline 11074.870. & 79.90 & 2.6 \\
\hline $11075.828 \ldots \ldots \ldots \ldots \ldots \ldots$ & -47.94 & 2.2 \\
\hline 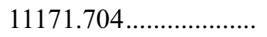 & -96.47 & 2.7 \\
\hline $11172.706 \ldots \ldots$ & 6.72 & 2.9 \\
\hline 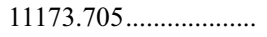 & 110.46 & 2.6 \\
\hline 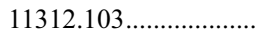 & -126.54 & 2.5 \\
\hline 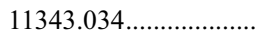 & -58.38 & 2.4 \\
\hline $11367.961 \ldots$ & -11.87 & 3.2 \\
\hline $11371.087 \ldots$ & -96.17 & 2.5 \\
\hline $11372.064 \ldots$ & -0.06 & 2.4 \\
\hline $11373.071 \ldots \ldots \ldots \ldots \ldots \ldots$ & 109.82 & 3.1 \\
\hline $11374.059 \ldots \ldots \ldots \ldots \ldots \ldots$ & 121.75 & 2.9 \\
\hline $11410.025 \ldots \ldots \ldots \ldots \ldots \ldots$ & 88.97 & 2.8 \\
\hline 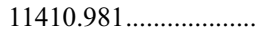 & -43.98 & 2.4 \\
\hline 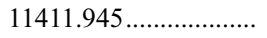 & -128.74 & 2.7 \\
\hline 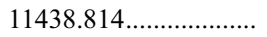 & 56.67 & 2.6 \\
\hline 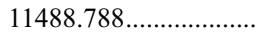 & 55.54 & 1.8 \\
\hline $11552.699 \ldots \ldots \ldots \ldots \ldots \ldots$ & 93.25 & 2.3 \\
\hline $11680.124 \ldots \ldots \ldots \ldots \ldots . .$. & 159.88 & 2.3 \\
\hline $11704.112 \ldots \ldots \ldots \ldots \ldots \ldots$ & -99.17 & 2.5 \\
\hline 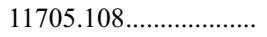 & -115.61 & 2.9 \\
\hline 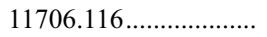 & -65.48 & 2.3 \\
\hline $11707.116 \ldots \ldots \ldots \ldots \ldots \ldots$ & 34.85 & 2.7 \\
\hline $11900.733 \ldots \ldots \ldots \ldots \ldots \ldots$ & 161.72 & 2.6 \\
\hline 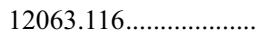 & 3.76 & 2.6 \\
\hline $12096.066 \ldots \ldots$ & -97.90 & 2.8 \\
\hline $12102.009 \ldots \ldots$ & 21.50 & 2.9 \\
\hline $12128.941 \ldots \ldots \ldots \ldots \ldots \ldots$ & 170.44 & 2.8 \\
\hline $12162.891 \ldots \ldots \ldots \ldots \ldots \ldots$ & 0.00 & 3.1 \\
\hline 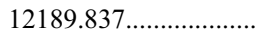 & -100.04 & 3.1 \\
\hline $12236.706 \ldots \ldots \ldots \ldots \ldots \ldots$ & 132.74 & 2.7 \\
\hline 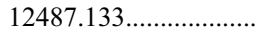 & -2.71 & 2.8 \\
\hline $12514.856 \ldots \ldots \ldots \ldots \ldots \ldots$ & 95.98 & 3.1 \\
\hline 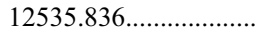 & 143.77 & 2.6 \\
\hline 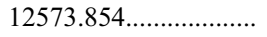 & -93.72 & 3.4 \\
\hline $12600.780 \ldots \ldots \ldots \ldots \ldots \ldots$ & 44.89 & 2.7 \\
\hline $12651.708 \ldots \ldots \ldots \ldots \ldots \ldots$ & -65.31 & 2.1 \\
\hline 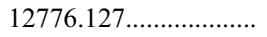 & 46.06 & 3.1 \\
\hline $12777.127 \ldots \ldots \ldots \ldots \ldots \ldots$ & 152.72 & 2.8 \\
\hline 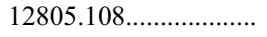 & 101.71 & 2.9 \\
\hline $12807.024 \ldots \ldots \ldots \ldots \ldots \ldots$ & 113.03 & 2.7 \\
\hline $12832.074 \ldots \ldots \ldots \ldots \ldots \ldots$ & -61.98 & 2.6 \\
\hline 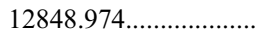 & 165.28 & 3.2 \\
\hline 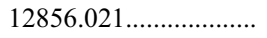 & 175.88 & 2.9 \\
\hline $12897.859 \ldots \ldots \ldots \ldots \ldots \ldots$ & 116.54 & 2.8 \\
\hline $12924.776 \ldots \ldots \ldots \ldots \ldots \ldots$ & -51.87 & 3.2 \\
\hline $12987.691 \ldots \ldots \ldots \ldots \ldots \ldots$ & -106.63 & 2.2 \\
\hline $13154.113 \ldots \ldots \ldots \ldots \ldots \ldots$ & 72.76 & 2.9 \\
\hline $13180.113 \ldots \ldots \ldots \ldots \ldots \ldots$ & -109.19 & 2.7 \\
\hline 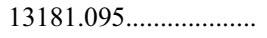 & -77.38 & 3.0 \\
\hline $13182.090 \ldots \ldots \ldots \ldots \ldots \ldots$ & 12.69 & 2.6 \\
\hline 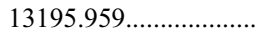 & -33.56 & 2.9 \\
\hline 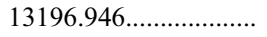 & 76.30 & 3.3 \\
\hline $13238.916 \ldots \ldots \ldots \ldots \ldots \ldots$ & -6.24 & 2.3 \\
\hline 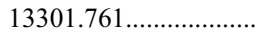 & -97.74 & 2.8 \\
\hline $13338.739 \ldots \ldots \ldots \ldots \ldots \ldots$ & 8.34 & 2.8 \\
\hline $13339.727 \ldots \ldots \ldots \ldots \ldots \ldots$ & 115.46 & 2.3 \\
\hline $13367.722 \ldots \ldots \ldots \ldots \ldots \ldots$ & 56.89 & 2.4 \\
\hline
\end{tabular}

\subsection{HD 37124}

HD 37124 (HIP 26381=GJ 209) has a spectral type G4 IV-V, a parallax of 30.1 mas (ESA 1997), and $M_{V}=5.07$ (Table 1). LTE analysis of our HIRES spectra shows that $[\mathrm{Fe} / \mathrm{H}]=-0.42$ (Valenti \& Fischer 2005; Fischer \& Valenti 2005), in good agreement with the value found by Santos et al. (2004) of $-0.38 \pm 0.04$ and by Laws et al. (2003) of $-0.37 \pm 0.03$. The $\mathrm{Ca}$ II $\mathrm{H}$ and $\mathrm{K}$ emission cores show that $\log R_{\mathrm{HK}}^{\prime}=-4.90$, implying an expected jitter of $3.2 \mathrm{~m} \mathrm{~s}^{-1}$ (Wright et al. 2004, $2005)$. From its $B-V$ of $0.67, M_{V}$, metallicity, and the models of Girardi et al. (2002), the stellar mass is $M=0.78 \pm 0.1 M_{\odot}$, in good agreement with the mass found by Santos et al. (2004) of $0.75 M_{\odot}$ and by Laws et al. (2003) of $0.77 M_{\odot}$. But see Fernandes \& Santos (2004), who find $M=0.94 M_{\odot}$.

The chromospheric $\log R_{\mathrm{HK}}^{\prime}$ implies a rotation period of 25 days and an age of $4 \pm 2$ Gyr, implying that this is a metal-poor star that is still on the main sequence (Wright 2004; Fernandes \& Santos 2004; Laws et al. 2003). Models of HD 37124 with a mass as high as $0.94 M_{\odot}$ can fit the observed stellar properties if a lower mixing length parameter of $\alpha=1.0$ instead of 1.7 (used in the Padova models) is adopted (Fernandes \& Santos 2004). Here we adopt a conventional lower mass of $0.78 M_{\odot}$, but we are struck by the need for improved interior physics of mainsequence stars on which the stellar mass and the companion $M \sin i$ depend.

The early velocities from Keck revealed an obvious Keplerian signal consistent with a planet having parameters $P=155.7$ days, $e=0.19, K=43 \mathrm{~m} \mathrm{~s}^{-1}$, and $M \sin i=1.0 M_{\mathrm{J}}$ (Vogt et al. 2000). Additional velocity measurements, a total of 30 spanning $5.4 \mathrm{yr}$, showed the one-planet model to be inadequate as seen in large residuals having rms $=13.3 \mathrm{~m} \mathrm{~s}^{-1}$ that were temporally correlated. We attempted to fit the velocities with a double-Keplerian model (Butler et al. 2003), the best of which contained the original planet, now with parameters $P=153.0$ days, $e=0.10$, and $M \sin i=0.86 M_{\mathrm{J}}$, and an additional planet having a long period of $\sim 6 \mathrm{yr}, e=0.75$, and $M \sin i=1.7 M_{\mathrm{J}}$.

However, $N$-body simulations showed that an eccentricity of 0.75 for the outer planet caused the system to disrupt within $10^{5} \mathrm{yr}$, much less than the star's age of $\sim 4$ Gyr (Butler et al. 2003). Goździewski (2003) carried out dynamical simulations covering longer timescales, finding that the outer planet was required to have $e<0.55$ for stability even if the system was coplanar. With the best-fit solution rendered dynamically unstable, the actual system has either an outer planet with lower eccentricity (within its uncertainty) or a qualitatively different structure.

We have now obtained a total of 52 velocity measurements spanning $8.4 \mathrm{yr}$ from Keck, warranting a new analysis. Table 7 gives the time, radial velocity, and internal error of each of the Doppler measurements. A periodogram of the velocities shows a strong peak at $P=154$ days with false alarm probability less than $0.1 \%$, supporting the original planet that dominates the velocity signal. A single-Keplerian model yields $P=154.5$ days, $K=35.6 \mathrm{~m} \mathrm{~s}^{-1}, e=0.14$, implying $M \sin i=0.88 M_{\mathrm{J}}$. Clearly, the original planet remains prominent in the new velocities, but the residuals still have a large scatter with $\mathrm{rms}=13.5 \mathrm{~m} \mathrm{~s}^{-1}$ and $\left(\chi_{\nu}^{2}\right)^{1 / 2}=2.62$, in comparison with internal errors of $3.2 \mathrm{~m} \mathrm{~s}^{-1}$ (median) and expected jitter of $3.2 \mathrm{~m} \mathrm{~s}^{-1}$. Thus, the singleKeplerian model still fails to explain the velocities.

In light of the instability of the previous two-planet fit, we searched for solutions that included a second orbiting companion. We examined the periodogram of the residuals to the single-Keplerian fit, which revealed peaks at periods of 31 , 

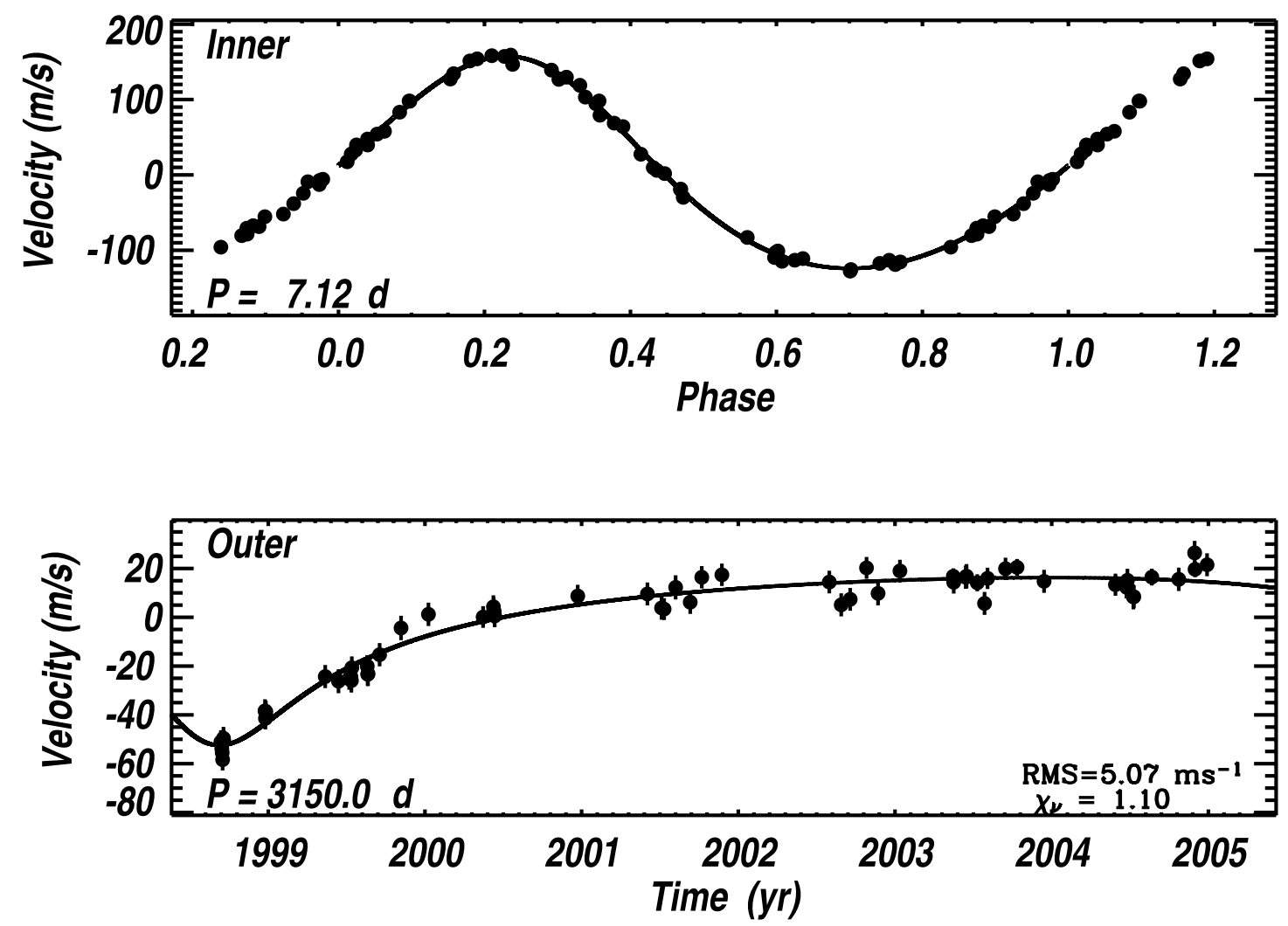

FIG. 5.-Measured velocities ( filled circles) and two-planet model (solid line) for HD 217107 from Keck. Top: Velocities vs. orbital phase for the inner planet with $M \sin i=1.4 M_{\mathrm{J}}, P=7.127$ days, and $e=0.13$, with the effects of the outer planet subtracted. Bottom: Velocities vs. time for the outer planet (inner planet subtracted), with an uncertain orbit not yet completed. The outer companion has a period greater than $7 \mathrm{yr}$, but the curvature suggests that the period is less than $30 \mathrm{yr}$ and that the eccentricity is $0.4-0.7$. The mass is likely $2 M_{\mathrm{J}}-10 M_{\mathrm{J}}$.
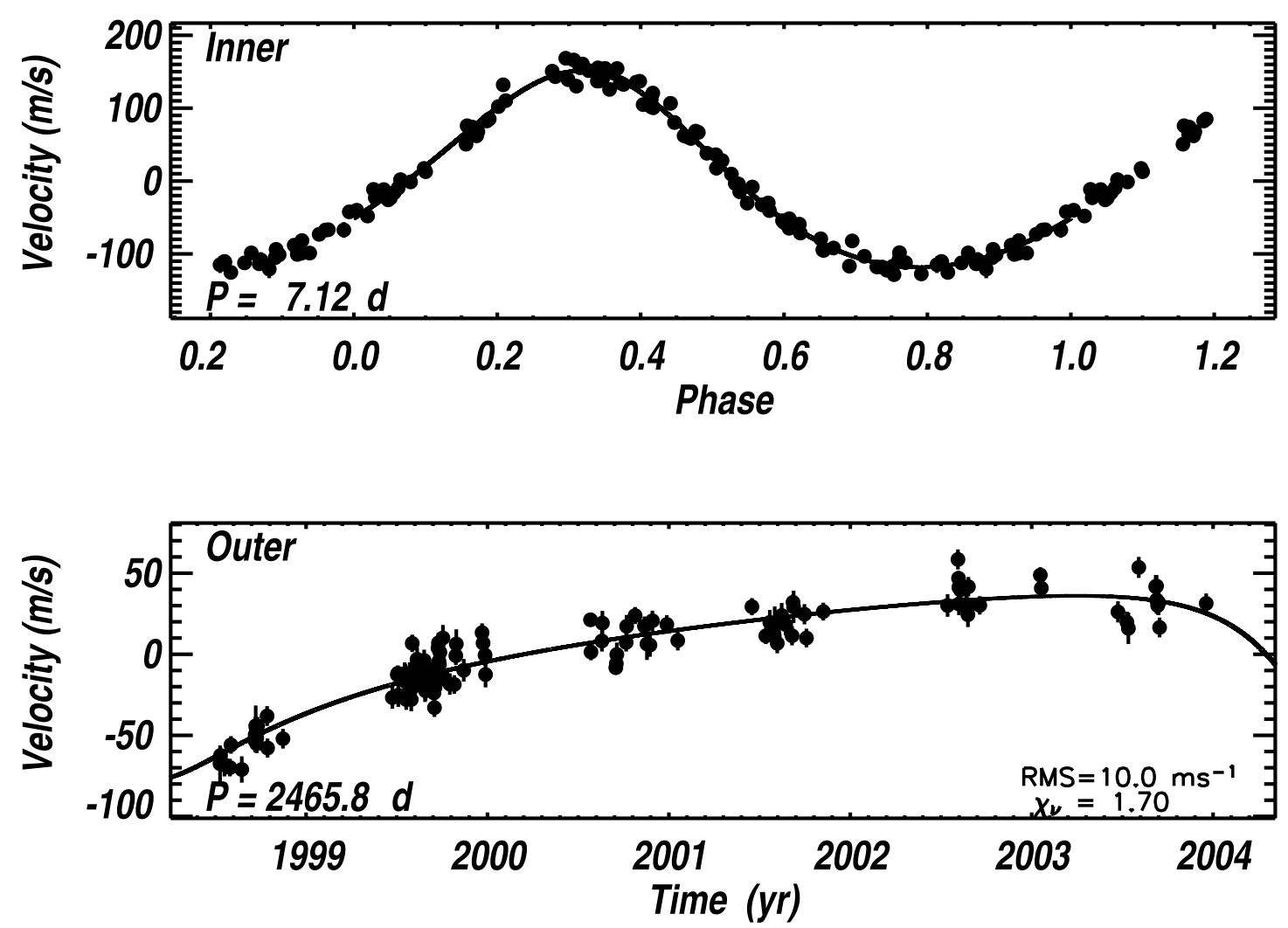

FIG. 6.- Same as Fig. 5, but all data from Lick Observatory. 
TABLE 6

Radial Velocities for HD 50499

\begin{tabular}{|c|c|c|}
\hline $\begin{array}{c}\text { JD } \\
(-2,440,000)\end{array}$ & $\begin{array}{c}\mathrm{RV} \\
\left(\mathrm{m} \mathrm{s}^{-1}\right)\end{array}$ & $\begin{array}{c}\text { Uncertainty } \\
\left(\mathrm{m} \mathrm{s}^{-1}\right)\end{array}$ \\
\hline $10418.985 \ldots$ & 15.93 & 3.3 \\
\hline $10462.944 \ldots$. & 8.08 & 2.0 \\
\hline $10546.793 \ldots \ldots \ldots \ldots \ldots \ldots \ldots \ldots . .$. & 8.89 & 3.7 \\
\hline 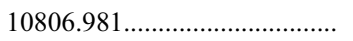 & 6.05 & 3.6 \\
\hline $10837.889 \ldots \ldots \ldots \ldots \ldots \ldots \ldots$ & -3.41 & 3.2 \\
\hline $10861.788 \ldots \ldots \ldots \ldots \ldots \ldots \ldots . .$. & 0.00 & 2.9 \\
\hline $11073.144 \ldots \ldots \ldots \ldots \ldots \ldots \ldots \ldots \ldots$ & -0.20 & 3.5 \\
\hline $11171.913 \ldots \ldots \ldots \ldots \ldots \ldots \ldots \ldots \ldots$ & 2.78 & 3.2 \\
\hline $11227.840 \ldots \ldots \ldots \ldots \ldots \ldots \ldots \ldots$ & 15.35 & 3.3 \\
\hline $11580.856 \ldots \ldots \ldots \ldots \ldots \ldots \ldots \ldots$ & 32.67 & 3.6 \\
\hline $11583.911 \ldots \ldots \ldots \ldots \ldots \ldots$. & 35.64 & 3.4 \\
\hline 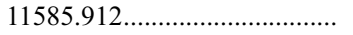 & 30.87 & 3.8 \\
\hline $11882.952 \ldots \ldots \ldots \ldots \ldots \ldots \ldots \ldots . .$. & 29.63 & 3.7 \\
\hline 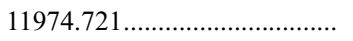 & 17.23 & 3.2 \\
\hline $12242.952 \ldots$ & 15.54 & 3.8 \\
\hline $12307.832 \ldots \ldots$ & 10.76 & 3.4 \\
\hline $12334.859 \ldots \ldots \ldots \ldots \ldots \ldots \ldots$ & 14.77 & 3.8 \\
\hline $12538.133 \ldots \ldots \ldots \ldots$. & 3.65 & 4.1 \\
\hline $12574.033 \ldots \ldots \ldots \ldots \ldots \ldots \ldots$ & 4.29 & 3.7 \\
\hline $12575.096 \ldots \ldots \ldots \ldots \ldots \ldots \ldots$. & 4.31 & 4.0 \\
\hline $12600.978 \ldots \ldots \ldots \ldots \ldots$ & -7.96 & 3.4 \\
\hline $12651.936 \ldots \ldots \ldots \ldots \ldots \ldots \ldots \ldots . .$. & -11.45 & 3.8 \\
\hline $12711.733 \ldots \ldots \ldots \ldots$ & -15.17 & 3.6 \\
\hline $12899.136 \ldots \ldots \ldots \ldots \ldots \ldots \ldots . .$. & -14.01 & 3.1 \\
\hline $12924.141 \ldots \ldots \ldots \ldots \ldots \ldots \ldots \ldots . .$. & -10.19 & 3.8 \\
\hline $12987.921 \ldots \ldots \ldots \ldots \ldots \ldots \ldots . .$. & -24.03 & 6.0 \\
\hline $12988.918 \ldots \ldots \ldots \ldots \ldots \ldots \ldots \ldots . .$. & -27.78 & 3.5 \\
\hline 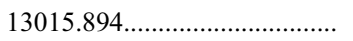 & -23.53 & 3.2 \\
\hline 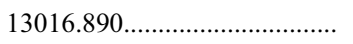 & -26.56 & 2.9 \\
\hline 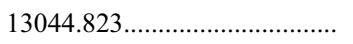 & -32.97 & 3.5 \\
\hline $13045.813 \ldots \ldots \ldots \ldots \ldots \ldots \ldots \ldots . .$. & -22.11 & 3.3 \\
\hline 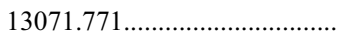 & -30.04 & 3.5 \\
\hline 13368.970 & -33.55 & 3.4 \\
\hline $13480.721 \ldots \ldots \ldots \ldots \ldots \ldots \ldots \ldots$ & -33.57 & 3.5 \\
\hline $13483.722 \ldots \ldots \ldots \ldots \ldots \ldots \ldots \ldots$ & -33.98 & 3.1 \\
\hline
\end{tabular}

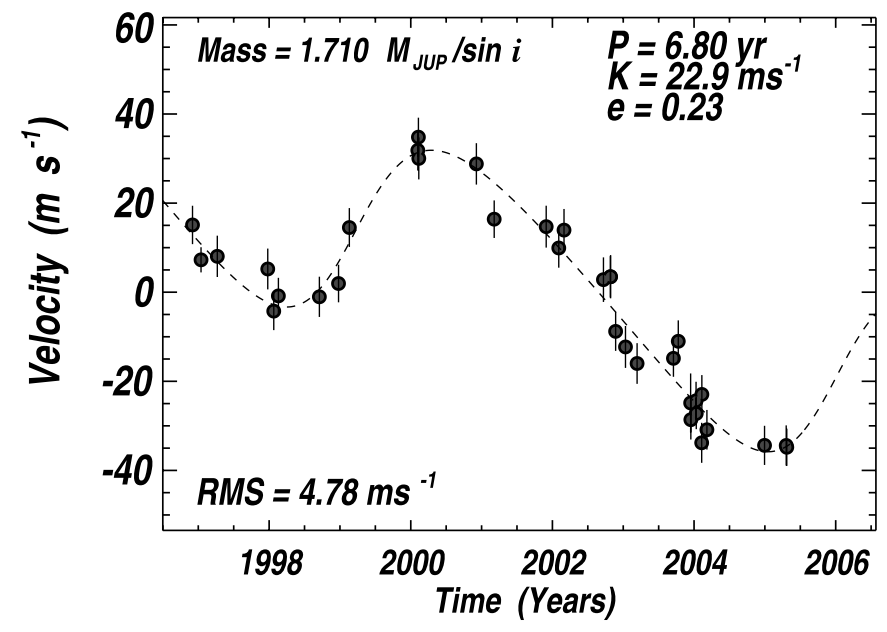

Fig. 7.-Measured velocity vs. orbital phase for HD 50499 ( filled circles) with the associated best-fit Keplerian including a linear trend. The orbital parameters are $P=6.8 \mathrm{yr}, K=22.9 \mathrm{~m} \mathrm{~s}^{-1}, e=0.23$, implying $M \sin i=1.71 M_{\mathrm{J}}$. The linear trend has slope $-4.8 \mathrm{~m} \mathrm{~s}^{-1} \mathrm{yr}^{-1}$, implying a second companion.
TABLE 7

Radial Velocities for HD 37124

\begin{tabular}{|c|c|c|}
\hline $\begin{array}{c}\text { JD } \\
(-2,440,000)\end{array}$ & $\begin{array}{c}\mathrm{RV} \\
\left(\mathrm{m} \mathrm{s}^{-1}\right)\end{array}$ & $\begin{array}{l}\text { Uncertainty } \\
\left(\mathrm{m} \mathrm{s}^{-1}\right)\end{array}$ \\
\hline $10420.047 \ldots .$. & 55.06 & 3.0 \\
\hline $10546.736 \ldots \ldots \ldots \ldots \ldots \ldots \ldots \ldots \ldots$ & 30.88 & 2.6 \\
\hline $10837.766 \ldots \ldots \ldots \ldots \ldots \ldots \ldots \ldots \ldots$ & 7.24 & 3.2 \\
\hline 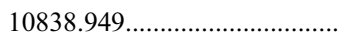 & 7.40 & 3.4 \\
\hline 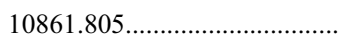 & 19.92 & 2.9 \\
\hline $11069.036 \ldots \ldots \ldots \ldots \ldots \ldots \ldots \ldots . .$. & -0.60 & 2.9 \\
\hline $11070.132 \ldots \ldots \ldots \ldots \ldots \ldots \ldots \ldots \ldots$ & 1.70 & 2.8 \\
\hline $11071.115 \ldots \ldots \ldots \ldots \ldots \ldots \ldots \ldots \ldots$ & 4.66 & 3.3 \\
\hline $11072.129 \ldots \ldots \ldots \ldots \ldots \ldots \ldots \ldots$ & -8.20 & 3.0 \\
\hline $11073.030 \ldots \ldots \ldots \ldots \ldots \ldots \ldots \ldots$ & -5.31 & 2.9 \\
\hline 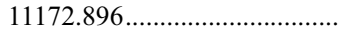 & 39.41 & 3.2 \\
\hline $11226.781 \ldots \ldots \ldots \ldots \ldots \ldots \ldots . .$. & 1.53 & 2.8 \\
\hline $11227.782 \ldots \ldots \ldots \ldots \ldots \ldots \ldots \ldots . .$. & 0.00 & 2.9 \\
\hline $11228.743 \ldots \ldots \ldots \ldots \ldots \ldots \ldots \ldots$ & -8.94 & 2.6 \\
\hline $11412.142 \ldots \ldots$ & -30.99 & 3.1 \\
\hline 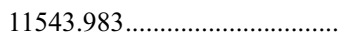 & -31.27 & 3.0 \\
\hline $11550.943 \ldots \ldots \ldots \ldots \ldots \ldots \ldots$ & -44.31 & 3.0 \\
\hline $11551.940 \ldots \ldots \ldots \ldots$ & -45.91 & 3.1 \\
\hline $11552.892 \ldots \ldots \ldots \ldots \ldots \ldots \ldots \ldots$ & -47.66 & 3.0 \\
\hline $11580.761 \ldots \ldots \ldots \ldots \ldots \ldots \ldots$ & -34.78 & 3.6 \\
\hline $11581.836 \ldots \ldots \ldots$ & -34.59 & 2.7 \\
\hline $11582.788 \ldots \ldots \ldots$ & -35.75 & 3.0 \\
\hline 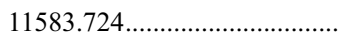 & -34.06 & 3.2 \\
\hline 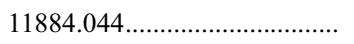 & -14.57 & 3.4 \\
\hline $11900.035 \ldots \ldots \ldots \ldots \ldots \ldots \ldots \ldots .$. & 3.00 & 2.8 \\
\hline $11974.800 \ldots \ldots \ldots \ldots \ldots \ldots \ldots \ldots . .$. & 42.08 & 2.9 \\
\hline $12007.745 \ldots \ldots \ldots \ldots \ldots \ldots \ldots \ldots$ & 6.63 & 3.2 \\
\hline $12242.991 \ldots \ldots \ldots \ldots \ldots \ldots \ldots \ldots \ldots$ & 48.57 & 3.1 \\
\hline $12333.945 \ldots \ldots \ldots \ldots \ldots \ldots \ldots \ldots$ & -16.70 & 3.5 \\
\hline $12334.786 \ldots \ldots \ldots \ldots \ldots \ldots \ldots . .$. & -10.42 & 3.6 \\
\hline $12536.128 \ldots \ldots \ldots \ldots \ldots \ldots \ldots \ldots$ & 14.01 & 3.5 \\
\hline $12537.086 \ldots \ldots \ldots \ldots \ldots \ldots \ldots \ldots \ldots$ & 13.67 & 3.7 \\
\hline $12573.038 \ldots \ldots \ldots \ldots \ldots \ldots \ldots$ & 29.57 & 3.4 \\
\hline $12574.999 \ldots \ldots \ldots \ldots \ldots \ldots \ldots \ldots \ldots$ & 29.58 & 3.6 \\
\hline $12576.022 \ldots \ldots \ldots \ldots \ldots \ldots \ldots$ & 25.62 & 3.5 \\
\hline $12601.000 \ldots \ldots \ldots \ldots \ldots \ldots \ldots \ldots . .$. & 8.73 & 3.5 \\
\hline $12602.032 \ldots \ldots \ldots \ldots \ldots \ldots \ldots \ldots .$. & 7.64 & 3.6 \\
\hline $12925.016 \ldots \ldots \ldots \ldots \ldots \ldots \ldots \ldots \ldots$ & 15.90 & 3.4 \\
\hline 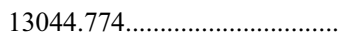 & 38.21 & 3.4 \\
\hline 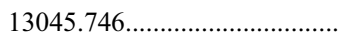 & 34.46 & 3.3 \\
\hline 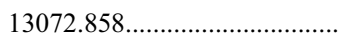 & -1.52 & 2.8 \\
\hline 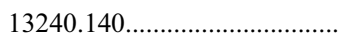 & -19.80 & 3.7 \\
\hline 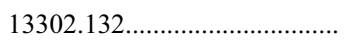 & -9.34 & 3.4 \\
\hline 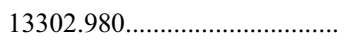 & -6.47 & 3.1 \\
\hline 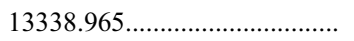 & 9.54 & 2.6 \\
\hline 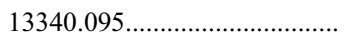 & 14.21 & 3.1 \\
\hline $13368.890 \ldots \ldots \ldots \ldots \ldots \ldots . .$. & -1.50 & 2.2 \\
\hline $13369.781 \ldots \ldots \ldots \ldots \ldots \ldots \ldots \ldots \ldots$ & -0.30 & 2.1 \\
\hline $13425.872 \ldots \ldots \ldots \ldots \ldots \ldots \ldots \ldots . .$. & -36.55 & 3.1 \\
\hline $13426.830 \ldots \ldots \ldots \ldots \ldots \ldots \ldots \ldots . .$. & -35.54 & 3.1 \\
\hline 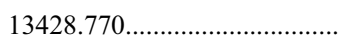 & -31.95 & 3.0 \\
\hline $13483.727 \ldots \ldots \ldots \ldots \ldots \ldots \ldots \ldots \ldots$. & 19.84 & 2.1 \\
\hline
\end{tabular}

$600-900$, and $\sim 2000$ days, the last of which corresponds to the second planet published in Butler et al. (2003). These three periods constituted the starting guesses for fits to models containing additional planets in the system. We also carried out a blind, panoramic search over a wide range of possible periods for an additional planet in the system. In both approaches we searched for a best-fitting double-Keplerian model using a Marquardt leastsquares minimization method in which the adopted uncertainty of each measurement was the quadrature sum of the internal 
velocity error (of each measurement) and the jitter. This panoramic search of parameter space achieved the lowest values of $\left(\chi_{\nu}^{2}\right)^{1 / 2}$ for periods of the second planet near 30, 870, and 2000 days, not surprisingly near the peaks of the periodogram.

We searched the parameter space of double-Keplerian models, composed of a planet with $P \sim 154$ days as the initial guess and those three prospective periods for the second orbiting companion. That original planet with $P \sim 154$ days always emerged from such attempts with a low eccentricity between 0.05 and 0.2 and $K \approx 30 \mathrm{~m} \mathrm{~s}^{-1}$, implying a minimum mass of $M \sin i=0.65 M_{\mathrm{J}}$ in a low-eccentricity orbit, similar to the single-Keplerian solution. Apparently, the reality and properties of this 154 day planet are robust, independent of the uncertain nature of additional companions. We found minima in $\left(\chi_{\nu}^{2}\right)^{1 / 2}$ and corresponding sets of the parameters $\left[P, K, e, \mathrm{rms},\left(\chi_{\nu}^{2}\right)^{1 / 2}\right]$ for the second companion as follows: (1909 days, $23.9 \mathrm{~m} \mathrm{~s}^{-1}$, $\left.0.85,8.1 \mathrm{~m} \mathrm{~s}^{-1}, 1.56\right),\left(31.05\right.$ days, $14.0 \mathrm{~m} \mathrm{~s}^{-1}, 0.48,9.45 \mathrm{~m} \mathrm{~s}^{-1}$, 1.83 ), and (876 days, $\left.16.6 \mathrm{~m} \mathrm{~s}^{-1}, 0.56,7.4 \mathrm{~m} \mathrm{~s}^{-1}, 1.45\right)$.

These three double-Keplerian models gave values of $\left(\chi_{\nu}^{2}\right)^{1 / 2}$ and rms (normalized for the degrees of freedom) that were significantly superior to the single-planet model, i.e., of smaller values. However, even the lowest value of $\left(\chi_{\nu}^{2}\right)^{1 / 2}$ of 1.45 suggests an inadequate fit, and the lowest rms of $7.4 \mathrm{~m} \mathrm{~s}^{-1}$ is similarly higher than can be explained by internal errors and jitter, both being $3.2 \mathrm{~m} \mathrm{~s}^{-1}$. Thus, none of the double-Keplerian models provide an adequate fit.

We searched for possible triple-Keplerian models, starting with two planets that had properties near the nearly successful double-Keplerian models and then adding an arbitrary third companion. We ran thousands of trials for different assumed parameters of the third planet, each trial employing a Marquardt least-squares algorithm. Two models emerged having $\left(\chi_{\nu}^{2}\right)^{1 / 2} \approx$ 1.0 and $\mathrm{rms}<5.5 \mathrm{~m} \mathrm{~s}^{-1}$, rendering both of them superior to the double-Keplerian models. [The value of $\left(\chi_{\nu}^{2}\right)^{1 / 2}$ is always computed with the degrees of freedom in the denominator, making $\left(\chi_{\nu}^{2}\right)^{1 / 2}$ a useful discriminate among models.] The topology of $\chi^{2}$ placed these two models in deep local minima, clearly superior to any other models. Other local minima had $\left(\chi_{\nu}^{2}\right)^{1 / 2} \sim$ 1.4 or more, clearly inferior. Both triple-planet models have one planet with $P \approx 154.5$ days and another planet with $P \approx$ 840 days. The two best models are distinguished by the third companion, having periods of either 2300 or 29.3 days yielding $\left(\chi_{\nu}^{2}\right)^{1 / 2}$ of 0.89 and 1.14 and rms of 3.86 and $5.12 \mathrm{~m} \mathrm{~s}^{-1}$, respectively.

To test stability of these two viable three-planet models, we carried out dynamical experiments of the three triple-planet models near their orbital periods and within their range of acceptable eccentricities from the uncertainties. The most promising system is the first, with three periods near $154.5,844$, and 2295 days. For eccentricities of the outer planet between 0.0 and 0.25 , the system is stable over $10^{5} \mathrm{yr}$ and has $\chi_{\nu}^{2}<1$. We consider all such models acceptable, and we cannot distinguish among them. In Table 2, we list the orbital parameters for all three planets of this triple-Keplerian model, labeled "model A," and we adopt $e=0.2$ (fixed) for the outer planet, a value within the dynamically stable solutions where the fit gives a low $\left(\chi_{\nu}^{2}\right)^{1 / 2}$ of 0.96 and $\mathrm{rms}=4.14 \mathrm{~m} \mathrm{~s}^{-1}$. This is a good fit, far superior to the two-planet fit and consistent with the errors and jitter. The eccentricity of the outer planet remains uncertain by \pm 0.1 .

Figure 8 shows the velocity measurements and the threeKeplerian fit to them for model A. In this model, the three planets have minimum masses $\left(M_{\mathrm{J}}\right)$, semimajor axes $(\mathrm{AU})$, and eccentricities $(M \sin i, a, e)$ of $(0.61,0.53,0.055),(0.60,1.64$, $0.14)$, and $(0.66,3.19,0.20$ - frozen). The three planets all have

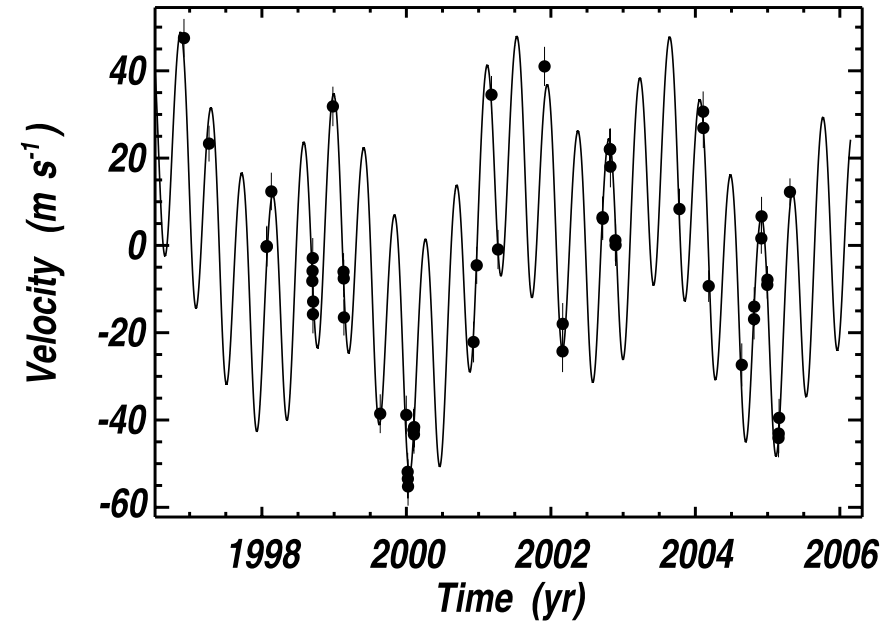

FIG. 8.-Measured velocities (filled circles) for HD 37124 vs. time. The solid line represents the best triple-Keplerian model A, one of two plausible solutions. This solution has three companions with minimum masses $(M \sin i)$ of $0.61 M_{\mathrm{J}}, 0.60 M_{\mathrm{J}}$, and $0.66 M_{\mathrm{J}}$ and periods of 154,844 , and 2295 days, respectively, all in low-eccentricity orbits.

minimum masses near $0.63 M_{\mathrm{J}} \pm 0.03 M_{\mathrm{J}}$, and all have low eccentricities, promoting stability.

The dynamical simulations of model A show that the three planets exchange eccentricities in a quasi-periodic manner. We carried out an eigenvalue analysis of the system's disturbing function and found that the eccentricity exchange observed in the dynamical simulations is well described by a three-planet secular theory that includes terms to second order in eccentricity (e.g., Murray \& Dermott 1999). Indeed, the full dynamical simulations show that all three planets maintain $e<0.3$ for apparently indefinite timescales. If the outer planet begins with $e=0$, the exchange of eccentricities limits the highest value of $e$ to be 0.15 .

This triple-planet model is clearly related to, and an improvement over, the double-Keplerian model presented in Butler et al. (2003). The outer planet now has $P=2295$ days and is identified with the second (outer) planet in the old model of Butler et al. (2003) that had $P \approx 6$ yr. That model was dynamically unstable, presenting a puzzle. In effect, that original double-Keplerian model has been augmented here with a middle planet having $P=843$ days, allowing the eccentricity of the outer planet to be lowered from 0.8 to 0.2 , and making the entire system stable. The apparent instability of the original system was probably caused by not including the middle planet.

However, our survey of triple-Keplerian parameter space revealed another domain, "model B," of good fits and dynamical stability. In this domain, the planets have periods near $29.3,154.7$, and 839.6 days. This is different from the model above in that the outer planet is replaced with an inner planet near $P=29$ days. For this solution, the three planets have values of minimum masses $\left(M_{\mathrm{J}}\right)$, semimajor axes (AU), and eccentricities $(M \sin i, a, e)$ of $(0.66,0.52,0.25),(0.73,1.60,0.15)$, and $(0.17$, $0.17,0.16)$. Here all three planets have modest eccentricities between 0.15 and 0.25 and the system is demonstrably stable in simulations covering $5 \times 10^{4} \mathrm{yr}$. This model B gives residuals having $\mathrm{rms}=5.1 \mathrm{~m} \mathrm{~s}^{-1}$ and $\left(\chi_{\nu}^{2}\right)^{1 / 2}=1.14$, clearly acceptable but worse than the fit for model A. In particular, the model A fit gave $\left(\chi_{\nu}^{2}\right)^{1 / 2}=0.96$. This model B three-planet fit has a lower probability of being correct both because of its lower $\left(\chi_{\nu}^{2}\right)^{1 / 2}$ and because the short-period inner planet brings a greater false alarm probability. Orbits of arbitrarily short periods can always be invoked to fit a time series. 
TABLE 8

Radial Velocities for HD 190360

\begin{tabular}{|c|c|c|}
\hline $\begin{array}{c}\text { JD } \\
(-2,440,000)\end{array}$ & $\begin{array}{c}\mathrm{RV} \\
\left(\mathrm{m} \mathrm{s}^{-1}\right)\end{array}$ & $\begin{array}{l}\text { Uncertainty } \\
\left(\mathrm{m} \mathrm{s}^{-1}\right)\end{array}$ \\
\hline $10365.721 \ldots$. & 18.83 & 2.4 \\
\hline $10605.059 \ldots \ldots \ldots \ldots \ldots \ldots \ldots . .$. & 30.15 & 2.2 \\
\hline $10666.910 \ldots$. & 17.70 & 2.1 \\
\hline 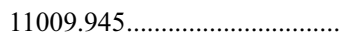 & -5.55 & 2.2 \\
\hline 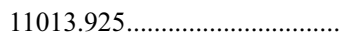 & -0.74 & 2.3 \\
\hline 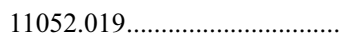 & 4.46 & 2.2 \\
\hline 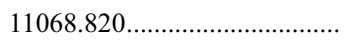 & -1.31 & 2.4 \\
\hline 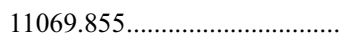 & 0.32 & 2.4 \\
\hline $11070.900 \ldots \ldots \ldots$ & 5.65 & 2.2 \\
\hline 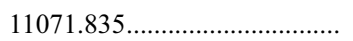 & -8.03 & 2.3 \\
\hline 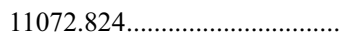 & -2.47 & 2.6 \\
\hline $11073.820 \ldots \ldots$ & -3.47 & 1.3 \\
\hline 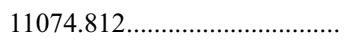 & -4.69 & 2.1 \\
\hline 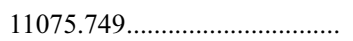 & -8.35 & 2.2 \\
\hline 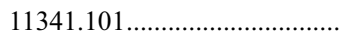 & -10.56 & 2.5 \\
\hline 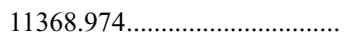 & -17.62 & 2.8 \\
\hline 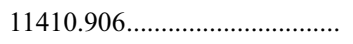 & -11.08 & 2.5 \\
\hline 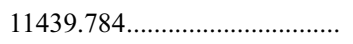 & -25.25 & 2.5 \\
\hline 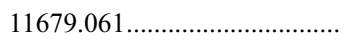 & -20.72 & 2.2 \\
\hline $11703.082 \ldots \ldots \ldots \ldots \ldots \ldots \ldots \ldots \ldots \ldots \ldots$ & -14.26 & 2.2 \\
\hline 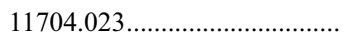 & -11.35 & 2.4 \\
\hline 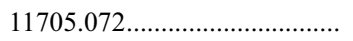 & -18.64 & 2.6 \\
\hline 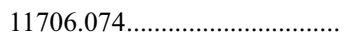 & -17.78 & 2.1 \\
\hline 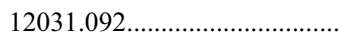 & -13.51 & 2.5 \\
\hline 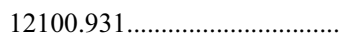 & -26.51 & 2.7 \\
\hline $12160.832 \ldots \ldots \ldots \ldots \ldots \ldots \ldots \ldots$ & -22.88 & 2.8 \\
\hline $12189.789 \ldots \ldots \ldots \ldots \ldots \ldots \ldots$ & -28.95 & 2.6 \\
\hline 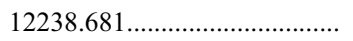 & -20.99 & 2.7 \\
\hline 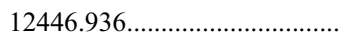 & -17.64 & 2.2 \\
\hline 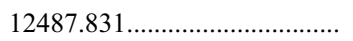 & -12.85 & 2.0 \\
\hline 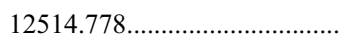 & -20.09 & 2.3 \\
\hline $12515.887 \ldots \ldots \ldots \ldots \ldots \ldots \ldots \ldots \ldots$ & -22.39 & 2.7 \\
\hline 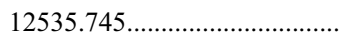 & -16.02 & 2.5 \\
\hline 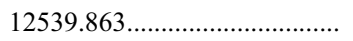 & -5.02 & 2.3 \\
\hline 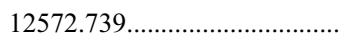 & -11.33 & 2.7 \\
\hline 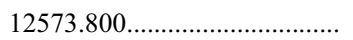 & -8.59 & 1.7 \\
\hline $12601.694 \ldots \ldots \ldots \ldots \ldots \ldots \ldots \ldots$ & -21.71 & 2.4 \\
\hline $12602.689 \ldots \ldots \ldots \ldots \ldots \ldots \ldots \ldots$ & -12.96 & 1.7 \\
\hline $12651.698 \ldots \ldots \ldots \ldots \ldots \ldots \ldots \ldots$ & -8.08 & 2.7 \\
\hline 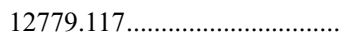 & -2.85 & 2.4 \\
\hline $12803.960 \ldots \ldots \ldots \ldots \ldots \ldots \ldots . .$. & -5.60 & 2.3 \\
\hline 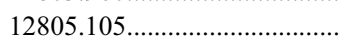 & -9.10 & 2.3 \\
\hline $12806.039 \ldots \ldots \ldots \ldots \ldots \ldots \ldots \ldots$ & -11.02 & 2.5 \\
\hline $12806.954 \ldots \ldots \ldots \ldots \ldots \ldots \ldots \ldots$ & -8.84 & 2.3 \\
\hline 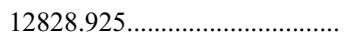 & -6.95 & 2.3 \\
\hline 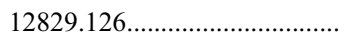 & -5.84 & 2.4 \\
\hline $12830.029 \ldots \ldots \ldots \ldots \ldots \ldots \ldots$ & -13.11 & 2.7 \\
\hline $12832.021 \ldots \ldots \ldots \ldots \ldots \ldots \ldots \ldots$ & -1.65 & 2.3 \\
\hline $12832.884 \ldots \ldots \ldots \ldots \ldots \ldots \ldots \ldots$ & -0.61 & 1.7 \\
\hline $12833.905 \ldots \ldots \ldots \ldots \ldots \ldots$ & -5.56 & 2.6 \\
\hline 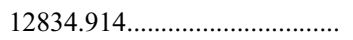 & -2.02 & 2.4 \\
\hline 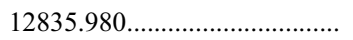 & -7.99 & 1.7 \\
\hline 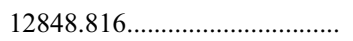 & 0.96 & 1.9 \\
\hline $12849.968 \ldots \ldots \ldots \ldots \ldots \ldots \ldots \ldots \ldots \ldots$ & 1.69 & 1.9 \\
\hline $12850.850 \ldots \ldots \ldots \ldots \ldots \ldots \ldots \ldots$ & 1.04 & 2.5 \\
\hline $12853.960 \ldots \ldots \ldots \ldots \ldots \ldots \ldots \ldots$ & -2.88 & 2.6 \\
\hline 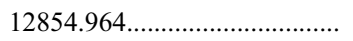 & -7.55 & 2.6 \\
\hline $12855.990 \ldots \ldots \ldots \ldots \ldots \ldots \ldots \ldots \ldots$ & -7.62 & 2.7 \\
\hline $12897.813 \ldots \ldots \ldots \ldots \ldots \ldots \ldots \ldots . .$. & 2.74 & 2.7 \\
\hline $12898.767 \ldots \ldots \ldots \ldots \ldots \ldots \ldots \ldots$ & 4.13 & 2.4 \\
\hline $12924.719 \ldots \ldots \ldots \ldots \ldots \ldots \ldots \ldots$ & -5.86 & 2.2 \\
\hline 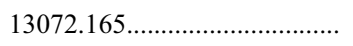 & 10.78 & 1.4 \\
\hline 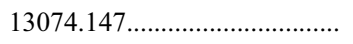 & 4.16 & 2.3 \\
\hline $13077.130 \ldots \ldots$ & -0.19 & 2.2 \\
\hline
\end{tabular}

TABLE 8-Continued

\begin{tabular}{|c|c|c|}
\hline $\begin{array}{c}\mathrm{JD} \\
(-2,440,000)\end{array}$ & $\begin{array}{c}\mathrm{RV} \\
\left(\mathrm{m} \mathrm{s}^{-1}\right)\end{array}$ & $\begin{array}{c}\text { Uncertainty } \\
\left(\mathrm{m} \mathrm{s}^{-1}\right)\end{array}$ \\
\hline $13154.110 \ldots \ldots \ldots$ & 14.39 & 2.4 \\
\hline 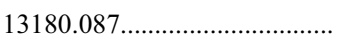 & 7.99 & 1.6 \\
\hline 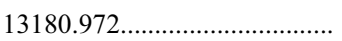 & 4.07 & 2.5 \\
\hline 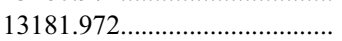 & 5.93 & 1.6 \\
\hline 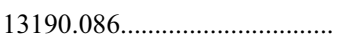 & 15.90 & 1.6 \\
\hline 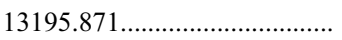 & 7.79 & 2.5 \\
\hline 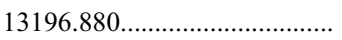 & 8.01 & 1.7 \\
\hline $13238.788 \ldots \ldots \ldots \ldots \ldots \ldots \ldots \ldots \ldots$ & 19.16 & 2.0 \\
\hline $13239.871 \ldots \ldots \ldots \ldots$ & 20.15 & 2.0 \\
\hline $13240.918 \ldots \ldots \ldots \ldots \ldots \ldots \ldots \ldots \ldots \ldots$ & 24.06 & 2.8 \\
\hline $13301.878 \ldots \ldots \ldots \ldots \ldots \ldots \ldots \ldots$ & 18.21 & 1.9 \\
\hline $13302.710 \ldots \ldots \ldots \ldots \ldots \ldots \ldots \ldots$ & 17.54 & 1.8 \\
\hline $13303.704 \ldots \ldots \ldots \ldots \ldots \ldots \ldots \ldots \ldots$ & 16.43 & 1.6 \\
\hline $13338.695 \ldots \ldots \ldots \ldots \ldots \ldots \ldots \ldots \ldots \ldots$ & 20.33 & 2.0 \\
\hline 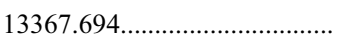 & 21.20 & 1.8 \\
\hline 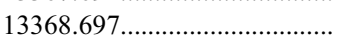 & 22.17 & 1.4 \\
\hline 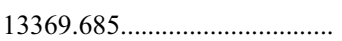 & 21.31 & 1.4 \\
\hline 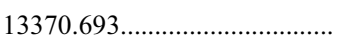 & 20.32 & 1.9 \\
\hline 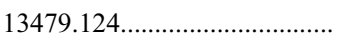 & 29.61 & 1.4 \\
\hline $13480.116 \ldots \ldots \ldots \ldots \ldots \ldots$ & 31.83 & 1.4 \\
\hline
\end{tabular}

In Table 2 we list both triple-planet solutions, labeling them model A and B, respectively. The system architecture of HD 37124 remains uncertain, as we cannot definitively distinguish between the two models. Additional velocity measurements for another few years are likely to clarify the orbital structure and masses of the planets in HD 37124. Velocity measurements by other velocity groups that can attain higher cadence may assess the reality of the 29 day period in model B.

\section{6. $H D 190360$}

The planet orbiting HD 190360 (=GJ 777 A=HIP 98767) was announced by the Geneva team to be the best "Jupiter analog," with a period of $2614 \pm 118$ days "on a quasi-circular orbit" (Udry et al. 2003). The authors described the importance of this planet as follows: "The long period and the almost zero eccentricity make the planet very similar to Jupiter" (Udry et al. 2003). The Geneva team emphasized the circular orbit and the lack of inward hot Jupiters as criteria for its status as a Jupiter analog (Kerr 2002). The reported minimum mass was $M \sin i=$ $1.15 M_{\mathrm{J}}$. We had been measuring the velocities of HD 190360 prior to the announcement of this planet but found a noncircular orbit for it, prompting us to continue observing it. A year later, the Geneva team published new orbital parameters and larger errors, $P=3902 \pm 1758$ days (Naef et al. 2003). However, here they reported that the eccentricity was either zero or $e=$ $0.48 \pm 0.2$ and that "a circular orbit cannot be excluded but it is rather unlikely." Their derived minimum mass was $1.33 M_{\mathrm{J}} \pm$ $0.19 M_{\mathrm{J}}$. The residuals to their fit were $9.3 \mathrm{~m} \mathrm{~s}^{-1}$, obtained with ELODIE and AFOE spectrographs.

HD 190360 has spectral type G6 IV and a parallax of 62.9 mas (ESA 1997). (Note a typographical error in the parallax in Naef et al. 2003.) It has $M_{V}=4.70$, placing it a few tenths of a magnitude above the main sequence (see Table 1), probably due to its high metallicity. Analysis of our HIRES spectra shows that $[\mathrm{Fe} / \mathrm{H}]=+0.21, T_{\text {eff }}=5551 \mathrm{~K}, V \sin i=2.2 \mathrm{~km} \mathrm{~s}^{-1}, \log R_{\mathrm{HK}}^{\prime}=$ -5.09 , and $P_{\text {rot }}=40$ days, implying an age of $7-10 \mathrm{Gyr}$ (Valenti $\&$ Fischer 2005; Wright et al. 2004). In comparison, Naef et al. (2003) reported $[\mathrm{Fe} / \mathrm{H}]=+0.21$ and $V \sin i<1 \mathrm{~km} \mathrm{~s}^{-1}$. The expected velocity jitter is $3.1 \mathrm{~m} \mathrm{~s}^{-1}$ (Wright et al. 2005). From 

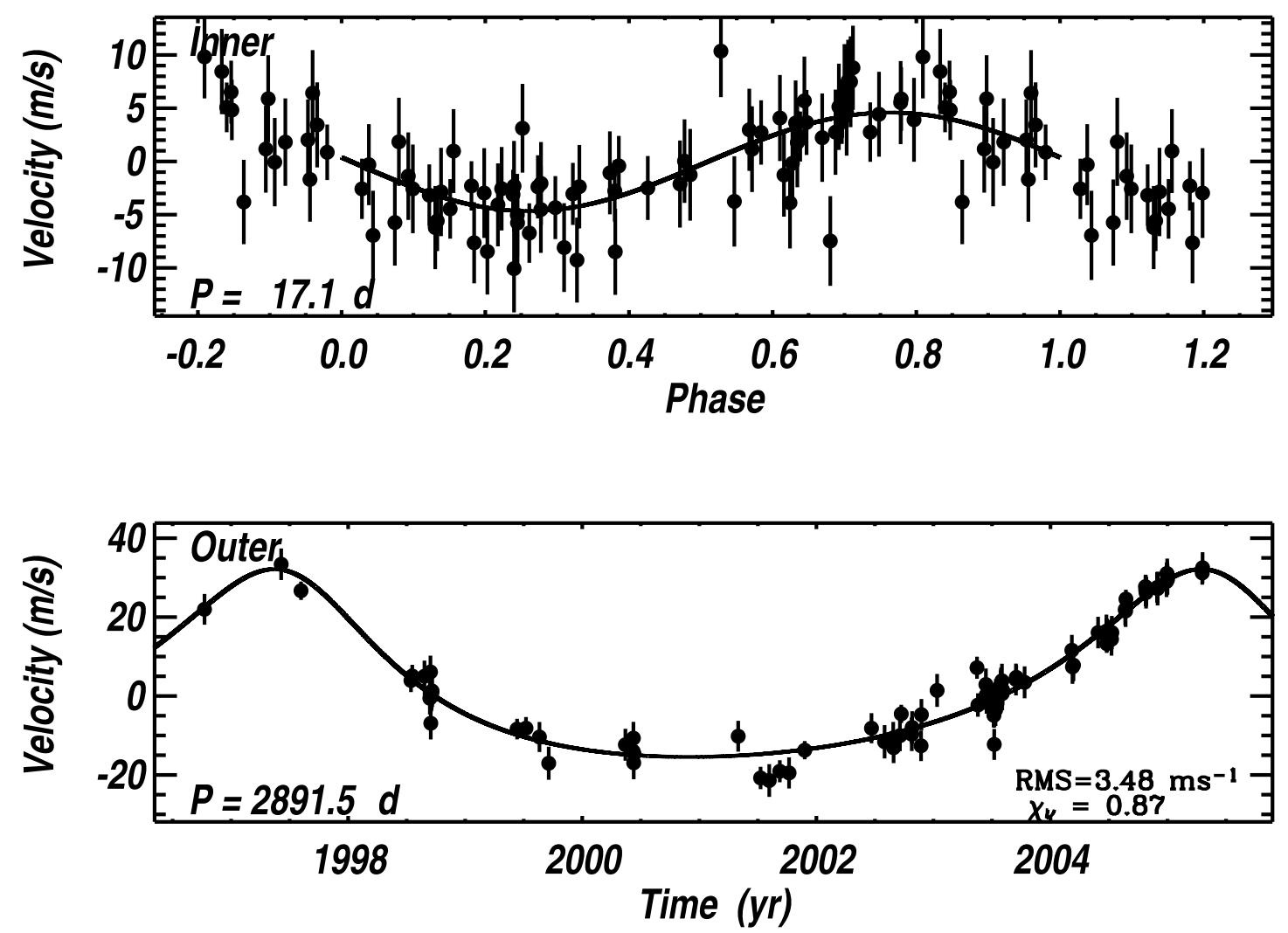

FIG. 9.-Measured velocities ( filled circles) and two-planet model (solid line) for HD 190360 from Keck. Top: Velocity vs. orbital phase for the inner planet with $P=17.1$ days, $e=0.01$, and $M \sin i=18.1 M_{\oplus}$, with the effects of the outer planet subtracted. Bottom: Velocity vs. time for the outer planet (inner planet subtracted), with $P=2891$ days, $e=0.36$, and $M \sin i=1.5 M_{\mathrm{J}}$. The residuals have $\mathrm{rms}=3.48 \mathrm{~m} \mathrm{~s}^{-1}$ and $\left(\chi_{\nu}^{2}\right)^{1 / 2}=0.87$ (including internal errors and jitter).

its $B-V$ of $0.72, M_{V}$, metallicity, and the models of Girardi et al. (2002), we find the stellar mass to be $M=0.96 \pm 0.1 M_{\odot}$, in agreement with the mass adopted by Naef et al. (2003).

Table 8 lists our 87 observations, including the times, measured velocities, and internal errors. A one-planet fit yields a period $P=2860$ days, $e=0.29$, and $M \sin i=1.3 M_{\mathrm{J}}$, giving residuals having an $\mathrm{rms}$ of $5.8 \mathrm{~m} \mathrm{~s}^{-1}$ and $\left(\chi_{\nu}^{2}\right)^{1 / 2}=1.35$. A periodogram of the residuals shows a strong peak at a period of 17.1 days with a false alarm probability of much less than $0.1 \%$, implying that the second periodicity is real.

Thus, the velocities demand inclusion of a second periodicity near 17.1 days. A model that invokes two planets yields lower residuals with an rms of $3.48 \mathrm{~m} \mathrm{~s}^{-1}$ and $\left(\chi_{\nu}^{2}\right)^{1 / 2}=0.88$, clearly a much better fit than with one planet. An $F$-test shows that the probability of $\left(\chi_{\nu}^{2}\right)^{1 / 2}$ dropping that much due to the inclusion of the second planet is less than $0.1 \%$ by mere fluctuations of noise. Therefore, the existence of the periodicity at 17.1 days is strongly supported.

Figure 9 shows the measured relative velocities and the associated best-fit double-Keplerian model. The current best fit gives, for the outer planet, $P_{b}=2891$ days, $e_{b}=0.36 \pm$ $0.03, K_{b}=23.5 \pm 0.5 \mathrm{~m} \mathrm{~s}^{-1}$, implying a minimum mass of $M_{b} \sin i_{b}=1.50 M_{\mathrm{J}}$. For the inner planet, the parameters are $P_{c}=17.10$ days, $e_{c}=0.01 \pm 0.1, K_{c}=4.6 \pm 1.1 \mathrm{~m} \mathrm{~s}^{-1}$, implying $M_{c} \sin i_{c}=0.057 M_{\mathrm{J}}=18.1 M_{\oplus}$.

The velocity semiamplitude of the inner planet, $K=4.6 \mathrm{~m}$ $\mathrm{s}^{-1}$, is small enough that one must consider the possibility that a stellar surface effect is the cause of the periodicity. From the chromospheric $\log R_{\mathrm{HK}}^{\prime}=-5.09$, we expect a rotation period of 36-44 days ( Wright et al. 2004), which is not consistent with the observed period of 17.1 days. Therefore, surface features are unlikely to be the cause of the 17.1 day Doppler period. One possibility is that the star has two spot complexes at opposite longitudes. However, this star is metal-rich, with $[\mathrm{Fe} / \mathrm{H}]=$ +0.25 , leading us to wonder if the calibration of rotation versus $\log R_{\mathrm{HK}}^{\prime}$ requires a modification due to metallicity. Metal-rich stars tend to exhibit low values of $\log R_{\mathrm{HK}}^{\prime}$ due to metallicity effects in the line and continuous opacity near $\mathrm{Ca}$ II $\mathrm{H}$ and $\mathrm{K}$ (Wright 2004). The velocity amplitude is too small for an analysis of line bisectors to reveal any surface kinematics as the cause.

Therefore, it remains possible that the 17.1 day velocity period is caused by the passage of features across the visible hemisphere of the rotating star. Unfortunately, we have not yet acquired any photometry of HD 190360. However, the most likely interpretation is that HD 190360 has an inner planet with $P=17.1$ days and $M \sin i=18.1 M_{\oplus}$.

\section{PHOTOMETRY OF THE HOST STARS}

We have obtained high-precision photometry of five of the six planetary host stars in this paper between 1998 October and 2004 December with the T8, T11, and T12 $0.8 \mathrm{~m}$ automatic photometric telescopes (APTs) at Fairborn Observatory. The APTs can detect short-term, low-amplitude brightness variations in the stars due to rotational modulation of the visibility of surface magnetic features (spots and plages), as well as longer term variations associated with stellar magnetic cycles (Henry 1999). The photometric observations thus help to establish whether observed radial velocity variations are caused by stellar activity or planetary reflex motion (e.g., Henry et al. 2000b). Queloz et al. (2001) and Paulson et al. (2004) have presented several examples of periodic radial velocity variations in solar-type stars caused 
TABLE 9

Photometric Results for the Planetary Host Stars

\begin{tabular}{|c|c|c|c|c|c|c|c|c|c|}
\hline $\begin{array}{l}\text { Star } \\
(1)\end{array}$ & $\begin{array}{c}\text { Planet } \\
\text { (2) }\end{array}$ & $\begin{array}{c}\text { Date Range } \\
\text { (HJD }-2,450,000) \\
(3)\end{array}$ & $\begin{array}{l}\text { Duration } \\
\text { (days) } \\
\text { (4) }\end{array}$ & $\begin{array}{c}N_{\text {obs }} \\
(5)\end{array}$ & $\begin{array}{c}\sigma \\
(\mathrm{mag}) \\
(6)\end{array}$ & $\begin{array}{l}\text { Planetary Period } \\
\text { (days) } \\
\text { (7) }\end{array}$ & $\begin{array}{l}\text { Semiamplitude } \\
\text { (mag) } \\
\text { (8) }\end{array}$ & $\begin{array}{c}\text { Transit Probability } \\
\qquad(\%) \\
(9)\end{array}$ & $\begin{array}{c}\text { Transits } \\
\text { (10) }\end{array}$ \\
\hline \multirow[t]{7}{*}{ 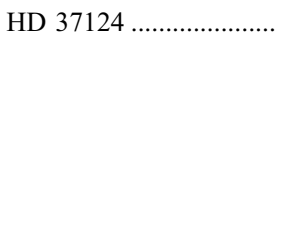 } & $\ldots$ & $1877-3378$ & 1501 & 412 & 0.0016 & & $\ldots$ & $\ldots$ & $\ldots$ \\
\hline & $\mathrm{b}^{\mathrm{a}}$ & $\ldots$ & $\ldots$ & $\ldots$ & $\ldots$ & 154.36 & $0.00035 \pm 0.00010$ & 1.0 & $?$ \\
\hline & $c^{a}$ & $\ldots$ & $\ldots$ & $\ldots$ & $\ldots$ & 880.5 & $0.00036 \pm 0.00010$ & 0.3 & $?$ \\
\hline & $\mathrm{d}^{\mathrm{a}}$ & $\ldots$ & $\ldots$ & $\ldots$ & $\ldots$ & 2065. & $0.00026 \pm 0.00011$ & 0.2 & $?$ \\
\hline & $\mathrm{b}^{\mathrm{b}}$ & $\ldots$ & $\ldots$ & $\ldots$ & $\ldots$ & 154.89 & $0.00034 \pm 0.00010$ & 1.4 & $?$ \\
\hline & $c^{b}$ & $\ldots$ & $\ldots$ & $\ldots$ & $\ldots$ & 29.33 & $0.00032 \pm 0.00010$ & 2.5 & No: \\
\hline & $\mathrm{d}^{\mathrm{b}}$ & $\ldots$ & $\ldots$ & $\ldots$ & $\ldots$ & 836.6 & $0.00036 \pm 0.00010$ & 0.4 & $?$ \\
\hline \multirow[t]{2}{*}{ 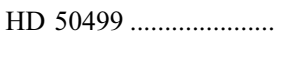 } & $\ldots$ & $2576-3087$ & 511 & 46 & 0.0030 & $\ldots$ & $\ldots$ & $\ldots$ & $\ldots$ \\
\hline & $\mathrm{b}$ & $\ldots$ & $\ldots$ & $\ldots$ & $\ldots$ & 3008.3 & $\mathrm{c}$ & 0.1 & $?$ \\
\hline \multirow[t]{3}{*}{ HD $108874 \ldots \ldots \ldots \ldots \ldots \ldots$} & $\ldots$ & $2599-3194$ & 595 & 274 & 0.0024 & $\ldots$ & $\ldots$ & $\ldots$ & $\ldots$ \\
\hline & $\mathrm{b}$ & $\ldots$ & $\ldots$ & $\ldots$ & $\ldots$ & 398.3 & $0.00077 \pm 0.00028^{\mathrm{d}}$ & 0.5 & No: \\
\hline & $\mathrm{c}$ & $\ldots$ & $\ldots$ & $\ldots$ & $\ldots$ & 1687.0 & $\mathrm{c}$ & 0.3 & $?$ \\
\hline \multirow[t]{3}{*}{ 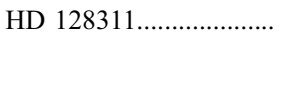 } & $\cdots$ & $3122-3194$ & 72 & 153 & 0.0081 & $\ldots$ & $\ldots$ & $\cdots$ & $\cdots$ \\
\hline & $\mathrm{b}$ & $\ldots$ & $\ldots$ & $\ldots$ & $\ldots$ & 455.9 & e & 0.4 & $?$ \\
\hline & $\mathrm{c}$ & $\ldots$ & $\ldots$ & $\ldots$ & $\ldots$ & 920.6 & e & 0.2 & $?$ \\
\hline \multirow[t]{3}{*}{ HD $190360 \ldots \ldots \ldots \ldots \ldots \ldots$} & $\ldots$ & $\ldots$ & $\ldots$ & $\ldots$ & $\ldots$ & $\ldots$ & $\ldots$ & $\ldots$ & $\ldots$ \\
\hline & $\mathrm{b}$ & $\ldots$ & $\ldots$ & $\ldots$ & $\ldots$ & 17.104 & f & 3.6 & $?$ \\
\hline & $\mathrm{c}$ & $\ldots$ & $\ldots$ & $\ldots$ & $\ldots$ & 2833.043 & $\mathrm{f}$ & 0.2 & $?$ \\
\hline \multirow[t]{4}{*}{ 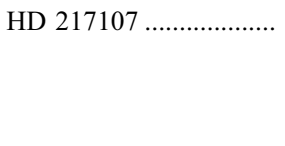 } & $\ldots$ & $1434-3364$ & 1930 & 373 & 0.0016 & $\ldots$ & $\ldots$ & $\ldots$ & $\ldots$ \\
\hline & $\mathrm{b}$ & $\ldots$ & $\ldots$ & $\ldots$ & $\ldots$ & 7.1269 & $0.00013 \pm 0.00011$ & 7.8 & No \\
\hline & $\mathrm{c}^{\mathrm{g}}$ & $\ldots$ & $\ldots$ & $\ldots$ & $\ldots$ & 2513.598 & $0.00012 \pm 0.00011^{\mathrm{h}}$ & 0.4 & $?$ \\
\hline & $\mathrm{c}^{\mathrm{i}}$ & $\ldots$ & $\ldots$ & $\cdots$ & $\ldots$ & 2465.891 & $0.00012 \pm 0.00011^{\mathrm{h}}$ & 0.3 & $?$ \\
\hline
\end{tabular}

a Model A. See $\S 3.5$.

b Model B. See $\S 3.5$.

c Duration of the photometric record is short relative to the planetary orbital period.

${ }^{d}$ Upper limit since slightly affected by PMT failures. See $\S 4$.

e Star is photometrically variable on the 11.53 day rotation period.

${ }^{\mathrm{f}}$ No photometric data on this star.

g Solution from Keck Observatory alone. See $\S 3.3$.

h Duration of the photometric record is somewhat shorter than one orbital cycle.

i Solution from Lick Observatory alone. See $\S 3.3$.

by photospheric spots and plages. The photometric observations are also useful to search for transits of the planetary companions (e.g., Henry et al. 2000a).

The T8, T11, and T12 APTs are all equipped with twochannel precision photometers employing two EMI 9124QB bi-alkali photomultiplier tubes to make simultaneous measurements in the Strömgren $b$ and $y$ passbands. The APTs measure the difference in brightness between a program star and a nearby constant comparison star. The automatic telescopes, photometers, observing procedures, and data reduction techniques are described in Henry (1999). Further details on the development and operation of the automated telescopes can be found in Henry (1995a, 1995b) and Eaton et al. (2003).

The Strömgren $b$ and $y$ differential magnitudes have been corrected for differential extinction with nightly extinction coefficients and transformed to the Strömgren system with yearly mean transformation coefficients. The external precision of the differential magnitudes is typically between 0.0012 and 0.0017 mag for these telescopes, as determined from observations of pairs of constant stars. The local comparison stars used for each program star were HD 37466 (for HD 37124), HD 47946 (for HD 50499), HD 109031 (for HD 108874), HD 127247 (for HD 128311), and HD 217131 (for HD 217107); all five comparison stars are intrinsically constant to $\sim 0.002$ mag or better, as determined by intercomparison with additional comparison stars. We combined the Strömgren $b$ and $y$ differential magnitudes into a single $(b+y) / 2$ passband to maximize the precision of the photometric measurements.
The photometric results for the five stars we measured are summarized in Table 9. We have no photometry as yet on HD 190360. Columns (4) and (5) give the duration of the photometric observations in days and the total number of individual differential magnitudes, respectively. The standard deviations in column (6) refer to the spread of the $(b+y) / 2$ measurements around the mean values of the data sets. The standard deviation of HD 50499 is somewhat elevated because of its southerly declination of $-34^{\circ}$ and the consequent high air mass of the observations. With the exception of HD 128311 (see below), all standard deviations are consistent with the constancy of the host stars. Periodogram analyses of the four constant stars do not reveal any significant periodicities. For all cases where the duration of the photometric observations is comparable to or longer than the radial velocity period, we compute the semiamplitudes of the light curves (col. [8]) with least-squares sine fits of the observations phased to the radial velocity periods. The resulting amplitudes are well below 0.001 mag for all cases that could be measured. The amplitude for HD 108874 is slightly affected by systematic errors resulting from degradation of the original photomultiplier tubes and their replacement with new tubes and so should be considered an upper limit. The photometric constancy of all the stars (except HD 128311) supports planetary reflex motion as the cause of the radial velocity variations.

Column (9) of Table 9 gives the geometric probability of transits for all of the planetary companions, computed from equation (1) of Seagroves et al. (2003) and assuming random orbital inclinations. Due to uncertainties in the orbital elements, 


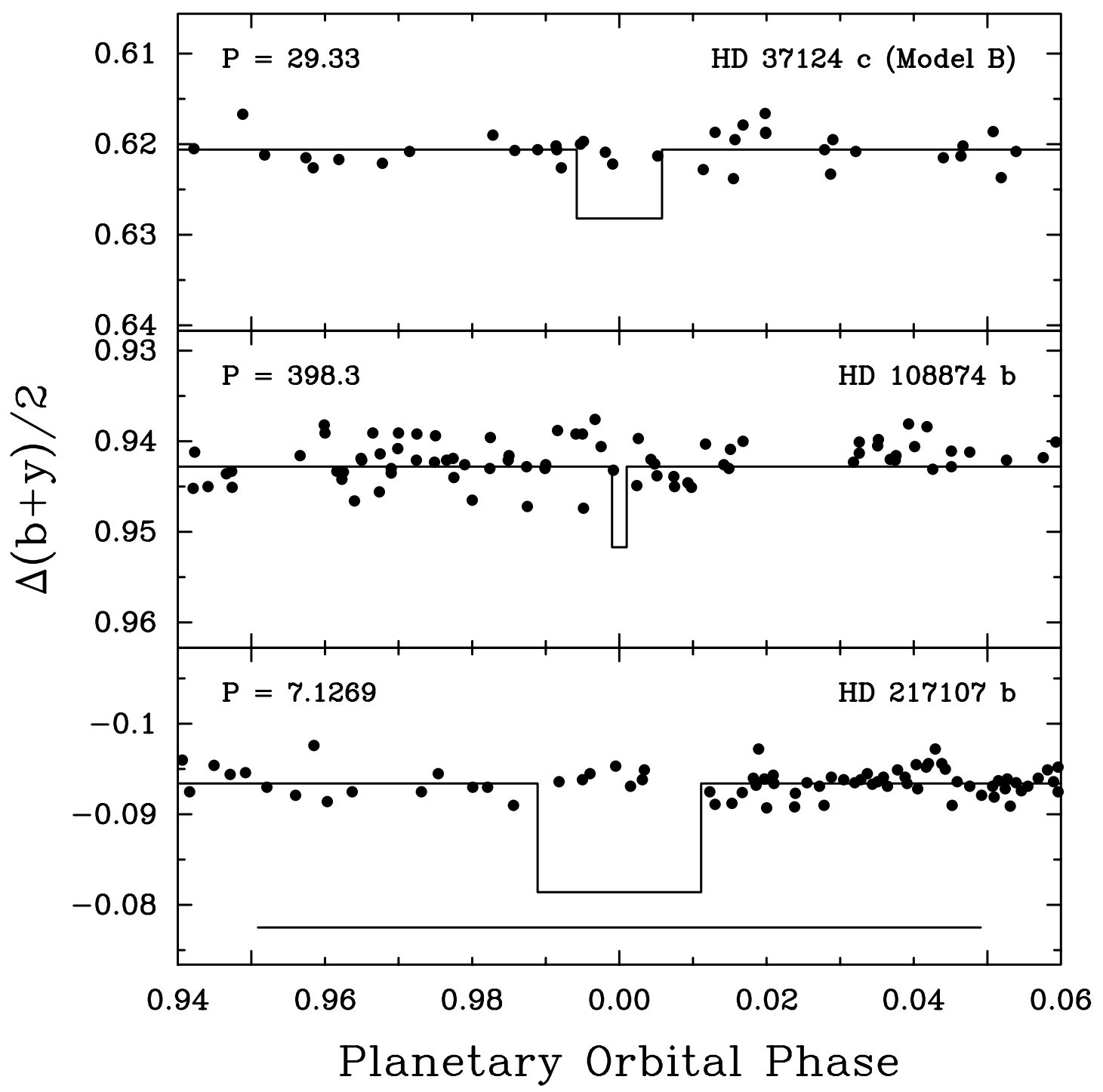

FIG. 10.-Photometric observations around the predicted times of transit for the three cases where effective transit searches were possible. Transits are largely ruled out for HD $37124 \mathrm{c}$ (top) in spite of a large uncertainty in the time of mid-transit. Numerous observations exist around the predicted transit time for HD $108874 \mathrm{~b}$ (middle), but transits remain possible due to small gaps in the phase coverage. Transits of HD $217107 \mathrm{~b}$ are ruled out (bottom) by their long predicted duration and the good phase coverage of the observations.

the predicted times of transit in many cases have error bars that are substantial fractions of an orbital period. Thus, given our current photometric coverage of these systems, searches for transits are still premature. However, transits can be largely or completely ruled out in three cases (col. [10]). These cases are shown in the three panels of Figure 10, where the combined $(b+y) / 2$ differential magnitudes of each star around the predicted time of transit are plotted against planetary orbital phase computed from the orbital elements in Table 2; zero phase in each case refers to a time of inferior conjunction (mid-transit). The solid line in each panel approximates the predicted transit light curve, assuming a planetary orbital inclination of $90^{\circ}$ (central transits). The out-of-transit light level corresponds to the mean brightness of the observations. The transit durations are calculated from the orbital elements, while the transit depths are derived from the estimated stellar radii and the planetary radii from the models of Bodenheimer et al. (2003). The horizontal bar below the predicted transit curve of HD 217107 represents the approximate $\pm 1 \sigma$ uncertainty in the time of midtransit, based on Monte Carlo simulations and the uncertainties in the orbital elements. No error bars are shown for the transit times of HD $37124 \mathrm{c}$ (model B) and HD $108874 \mathrm{~b}$, since the uncertainties are larger than the width of the plot.

In spite of the large uncertainty in the time of mid-transit for HD $37124 \mathrm{c}$ (model B), the duration of the transit window (0.012 phase units), along with the average density of observations on the phase curve, predicts that approximately five observations should fall at random within the transit window regardless of its location in the phase curve. The lack of any clusters of low points anywhere in the phase curve makes the existence of transits of HD 37124 c very unlikely. The phase curve of HD 108874 at the phases of possible transits is similarly well covered. However, the predicted duration for transits of HD $108874 \mathrm{~b}$ is much shorter ( 0.002 phase units); this implies that only about 0.75 observations should fall at random within the transit window. No observations in transit are seen, but small gaps in the phase curve mean that transits cannot be completely ruled out. Transits are ruled out for HD $217107 \mathrm{~b}$, given the predicted depth and duration of the transits and the density of observations in the phase curve around the predicted time of mid-transit.

Finally, we look at our photometry of HD 128311. The observations span only 72 days, far shorter than the velocity periods 

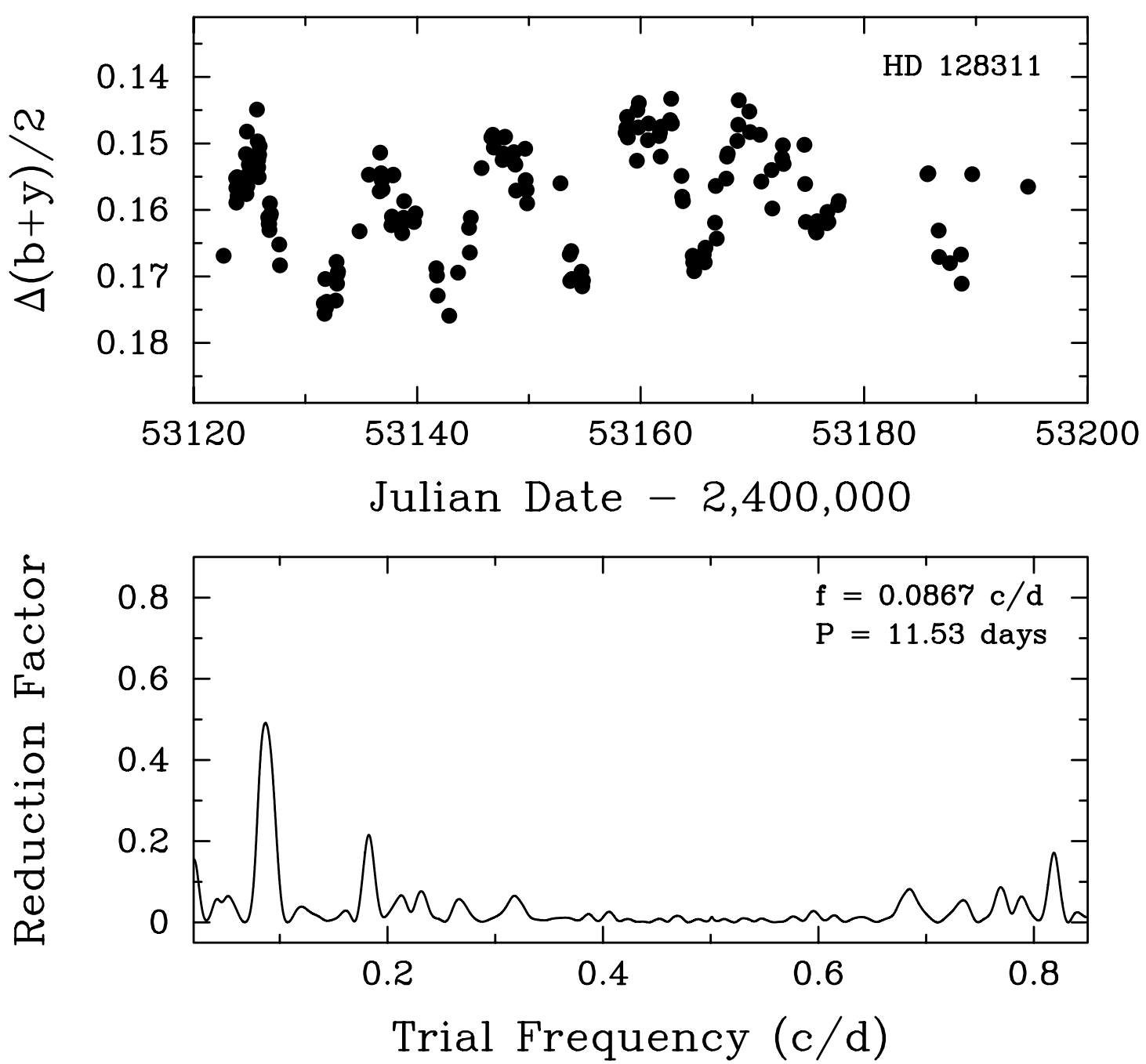

Fig. 11.-Photometric observations of HD 128311 in the top panel reveal cyclic brightness variations due to starspots with an amplitude of about 0.03 mag. Period analysis in the bottom panel gives the stellar rotation period of $11.53 \pm 0.15$ days. The rotation period is much shorter than the orbital period of either companion.

of the two companions, and have a standard deviation about their mean of $0.0081 \mathrm{mag}$ ( Table 9), far larger than the typical APT precision. The observations are plotted against Julian Date in the top panel of Figure 11 and show obvious low-amplitude ( $\sim 0.03 \mathrm{mag}$ ) variability due to starspots. A power spectrum of the observations is shown in the bottom panel of Figure 11, computed with the method of Vaníček (1971), and gives a period of $11.53 \pm 0.15$ days, which we interpret as the stellar rotation period. This confirms the photometric period reported by Strassmeier et al. (2000). As discussed in $\S 3.1$, the velocity residuals to the two-planet fit exhibit strong periodicity at 5.6 days, half the stellar rotation period, suggesting that the velocity residuals arise from spots existing at times on opposite hemispheres of the star. Indeed, the last couple of cycles of our photometry indicate that a new secondary minimum may be evolving on the descending branch of the light curve. Although the star is photometrically variable, the long-period velocity variations are clearly not the result of rotational modulation of spots.

\section{DYNAMICAL INTERACTIONS OF HD 128311 AND HD 108874}

\section{1. $H D 128311$}

All of our orbital fits in Table 2 use Keplerian ellipses as fitting functions; the assumption of purely elliptical orbits implies that the gravitational interactions between the planets are negligible, and indeed this is an excellent approximation over the $\tau<10 \mathrm{yr}$ span of radial velocity observations. For the HD 128311 system, the velocity jitter of $20 \mathrm{~m} \mathrm{~s}^{-1}$ of the parent star allows for a wide range of planetary configurations that fit the observed velocities. In order to connect a pair of elliptical orbits to a particular threebody initial condition, one must specify an epoch at which the Keplerian parameters are to be interpreted as osculating orbital elements. Given an epoch, one can integrate the system forward to investigate its dynamics and long-term stability. When we do this for HD $128311 \mathrm{~b}$ and c, we find that the best-fit two-Keplerian system listed in Table 2 suffers a close encounter between the planets within $2000 \mathrm{yr}$ for all of the epochs that we have tested.

Using the self-consistent fitting code described in Laughlin et al. (2005), which employs three-body initial conditions rather than Keplerian ellipses to model the radial velocities, we can obtain alternate two-planet fits to the HD 128311 data set that also yield $\left(\chi_{\nu}^{2}\right)^{1 / 2} \sim 1$ (given the $\sigma=9 \mathrm{~m} \mathrm{~s}^{-1}$ jitter value for the star). For example, our best-guess self-consistent model of the HD 128311 radial velocities has $P_{b}=464.9 \pm 4.9$ days, $P_{c}=909.6 \pm$ 11.0 days, $T_{p_{b}}=\mathrm{JD} 2,450,650.34, T_{p_{c}}=\mathrm{JD} 2,450,563.49, e_{b}=$ $0.38 \pm 0.08, e_{c}=0.21 \pm 0.07, \varpi_{b}=80^{\circ} .1 \pm 16^{\circ}, \varpi_{c}=21^{\circ} .6 \pm$ $61^{\circ}, M_{b}=1.56 M_{\mathrm{J}} \pm 0.16 M_{\mathrm{J}}$, and $M_{c}=3.08 M_{\mathrm{J}} \pm 0.11 M_{\mathrm{J}}$.

This model yields an rms scatter of $14.7 \mathrm{~m} \mathrm{~s}^{-1}$. The system is indefinitely stable, due to the presence of a protective $2: 1$ 


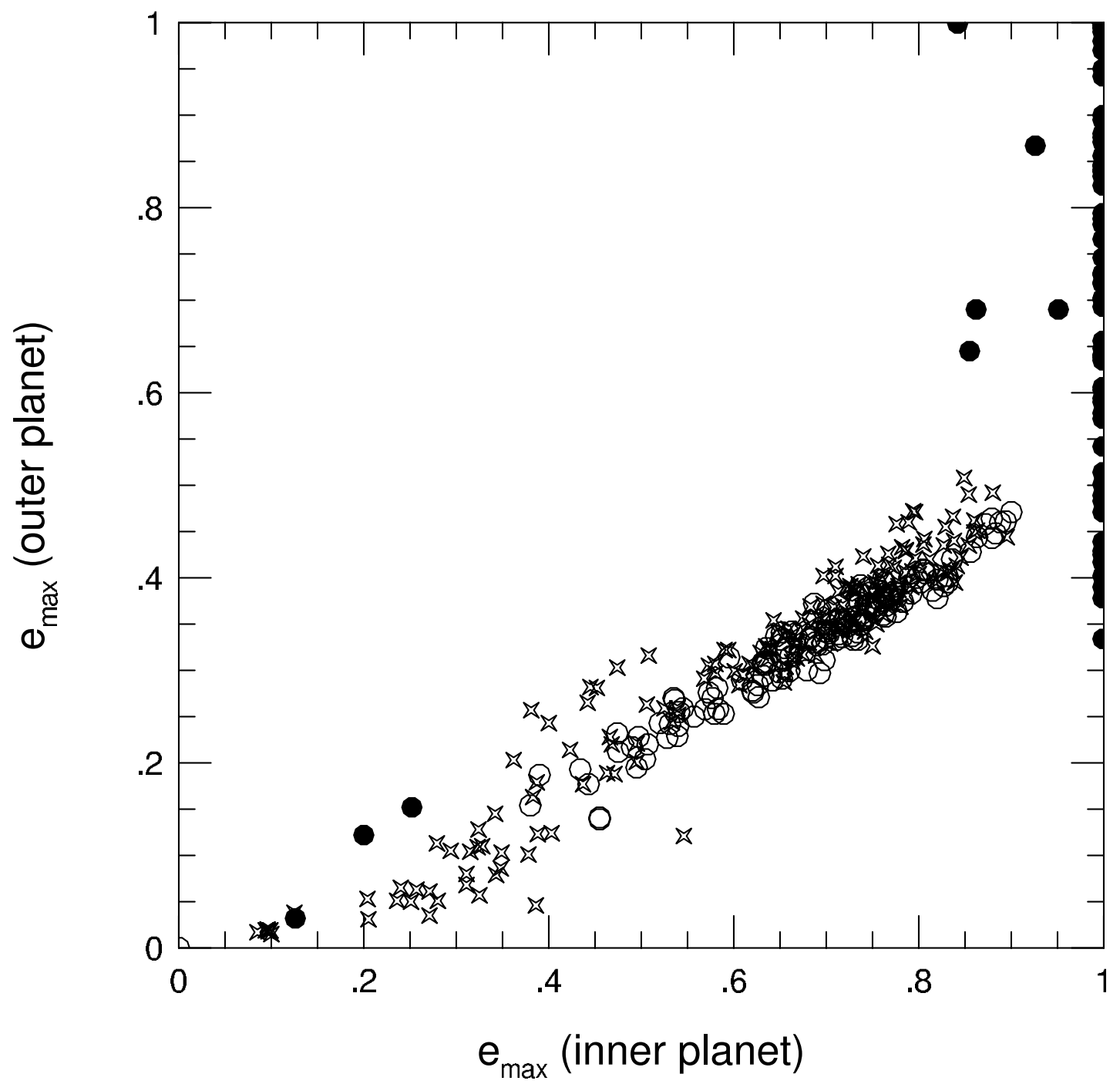

FIG. 12.-Maximum osculating eccentricities observed during $10^{4} \mathrm{yr}$ dynamical integrations of self-consistent fits obtained using individual bootstrap Monte Carlo realizations of the HD 128311 radial velocity data set. Open circles: Scenarios in which the $2: 1$ resonant arguments $\theta_{1}$ and $\theta_{2}$ both librate throughout a $10^{4}$ yr integration (130 scenarios, all stable). Stars: Scenarios in which $\theta_{1}$ librates and $\theta_{2}$ circulates throughout a $10^{4} \mathrm{yr}$ integration (188 scenarios, all stable). Filled circles: Scenarios in which $\theta_{1}$ and $\theta_{2}$ both circulate ( 409 scenarios, only 3 stable over $10^{4} \mathrm{yr}$ ).

resonance in which the argument $\theta_{2}=\lambda_{1}-2 \lambda_{2}$ is librating with a half-amplitude $\Delta \theta_{2}=60^{\circ}$. The startling difference in dynamical behavior between this fit and the fit listed in Table 2 stems from the fact that the relatively high stellar jitter and the long-term importance of planet-planet interactions lead to a situation where there are a variety of equally likely yet dynamically distinct solutions to the current radial velocity data set. Given the long orbital periods in the system, it is likely to be a long time before a substantially better determination of the orbits can be made on the basis of radial velocity fitting alone.

As we discuss further in an upcoming paper, however, the HD 128311 planetary system can be usefully constrained when dynamical stability integrations are coupled to the process of determining the uncertainty in the orbital fit. As a brief illustration, we take the self-consistent two-planet model given in the preceding paragraph and apply a bootstrap Monte Carlo procedure, in which alternate radial velocity data sets are generated by scrambling the residuals to the fit and then adding them back (with replacement) to the model velocity curve. We then use Levenberg-Marquardt minimization to generate a self-consistent fit to each of the Monte Carlo-generated data sets (adopting an unbiased initial guess with $P_{b_{i}}=450$ days, $P_{c_{i}}=900$ days, $M_{b_{i}}=2.0 M_{\mathrm{J}}, M_{c_{i}}=3.0 M_{\mathrm{J}}$, and $e_{b_{i}}=e_{c_{i}}=0$ ).

When a trial has converged, the resulting system is integrated for $10^{4} \mathrm{yr}$, and the maximum eccentricity attained by each planet during the integration is noted. Orbital instability manifests itself through the eccentricity of either planet approaching $e=1$. We also monitor the maximum excursions of the $2: 1$ resonant arguments $\theta_{1}$ and $\theta_{2}$ in order to determine whether each particular fit is in $2: 1$ resonance for the entire $10^{4} \mathrm{yr}$.

The results of this analysis are shown in Figure 12. In 727 Monte Carlo trials, we find that in only 3 out of 409 scenarios is the system both stable for 10,000 yr and not in resonance. Among the remaining 318 resonant cases, all have $\theta_{1}$ librating, while in addition, among these, $\theta_{2}$ is also librating in 130 instances. We can thus conclude, with a confidence of approximately $99 \%$, that the HD 128311 system is indeed stabilized by a 2:1 mean motion resonance.

\section{2. $H D 108874$}

The dual-Keplerian orbital fit listed in Table 2 for HD $108874 \mathrm{~b}$ and $\mathrm{c}$ is very dynamically active over a timescale that is much 
longer than the duration of radial velocity observations. For example, when an integration is started at epoch JD 2,451,340.806, the two planets maintain a secular Laplace-Lagrange mode of angular momentum exchange (e.g., Chiang et al. 2001). In other trials, however, the resulting oscillations proceed to instability, leading to destruction of the system on a timescale of about $500,000 \mathrm{yr}$. The evolution is highly chaotic and thus sensitively dependent on initial conditions.

We find, however, that dynamically stable, self-consistent fits are readily obtained using Levenberg-Marquardt minimization when the Keplerian orbital parameters given in Table 2 are used as an initial guess. For example, a stabilized fit referenced to epoch JD 2,451,340.806 has $P_{b}=394.294$ days, $P_{c}=1600.099$ days, $T_{p_{b}}=\mathrm{JD} 2,451,322.74, T_{p_{c}}=\mathrm{JD} 2,450,591.33, e_{b}=0.045$, $e_{c}=0.000, \varpi_{b}=255^{\circ} .46, \varpi_{c}=252^{\circ} .18, M_{b}=1.223 M_{\mathrm{J}}$, and $M_{c}=1.022 M_{\mathrm{J}}$ and appears to be indefinitely stable. The orbits of the two planets are close to the $4: 1$ commensurability, but for these particular initial conditions, none of the four $4: 1$ resonant angles are librating. This situation is to be expected because $4: 1$ resonance is very narrow at low eccentricity (Murray \& Dermott 1999). As was also the case with the planets orbiting HD 128311 , the HD 108874 system can currently be described by a large number of dynamically distinct configurations that are fully consistent with the radial velocity data.

\section{DISCUSSION}

The six systems presented here add to the growing inventory and diversity of multiple-component systems. Two of the systems, namely, HD 217107 and HD 190360, are distinctly "hierarchical," having ratios of orbital periods greater than 5, leading to weak interactions. Three of the systems, namely, HD 128311, HD 108874 , and HD 37124, are interactive, exchanging energy and angular momentum between the components that change the instantaneous orbital parameters on timescales much shorter than the age of the star. These three systems require theoretical calculations of their dynamics to understand their origin and their subsequent evolution.

Four of the six multicomponent systems discussed here, namely, HD 128311, HD 108874, HD 37124, and HD 190360, are almost certainly composed entirely of companions having masses less than $13 M_{\mathrm{J}}$, making them "planetary" by the commonly adopted (by IAU) mass threshold. Including these new planetary systems, a total of 17 multiplanet systems are now known. (HD 37124 was already known to be multiplanet.) As $\sim 135$ nearby stars are currently known to harbor planets, systems containing multiple giant planets, of Jupiter or Saturn mass, are apparently a common phenomenon. The fraction of known planet-bearing stars that contain multiple giant planets is roughly $17 / 135=0.13$.

Two of the systems, HD 217107 and HD 50499, have outer companions of such long period that their semimajor axis and mass remain poorly constrained. These outer components are likely to be planetary, but the possibility of very long periods and large orbital eccentricities leaves open the chance that they have masses greater than $13 M_{\mathrm{J}}$.

Of particular interest are resonance systems that offer fossil evidence of the migrational evolution and capture that established their mutual lock. Here we have added the apparent 2:1 resonance system HD 128311, making it the third such system, along with those in GJ 876 and HD 82943. It is certainly remarkable that 3 of 17 multiplanet systems reside in deep 2:1 resonances. Some combination of planet-disk interactions and planet-planet interactions must occur commonly during the early evolution of multiplanet systems to explain this high oc- currence of 2:1 resonances. Moreover, several other systems may be in, or near, higher order mean motion or secular resonances, including Upsilon And, 55 Cnc, HD 12661, and HD 202206. The system presented here, HD 108874, requires dynamical calculations to explore a possible $4: 1$ resonance. Thus, the migration and dynamical evolution of planetary systems commonly result in resonance capture, with the actual scenario depending presumably on initial conditions and on the properties of the disk (Bryden et al. 2000; Rivera \& Lissauer 2001; Laughlin \& Chambers 2001; Lee \& Peale 2002; Chiang \& Murray 2002; Ida \& Lin 2004).

HD 37124 is a remarkable planetary system in many respects. Our analysis suggests that it contains three planets having $M \sin i \approx 0.65 M_{\mathrm{J}}$. We present two plausible models for those three planets, one with an outer planet having a period of $6 \mathrm{yr}$ and another with a short-period planet of only 29 days. We favor the former but cannot rule out the latter. This system around HD 37124 is the fourth known triple-planet system around a nearby star, joining 55 Cnc, Upsilon And, and HD 160691. The star itself has subsolar metallicity. Three groups find $[\mathrm{Fe} / \mathrm{H}]=$ -0.4 , making this star one of the most metal-poor known among planet-bearing stars (Santos et al. 2004; Fischer \& Valenti 2005). As planet formation in the core accretion model is enhanced by high metallicity, this metal-poor star that harbors three giant planets certainly poses a major puzzle. Why should such a metal-poor star harbor more giant planets than the vast majority of G0 V stars that have solar metallicity or more?

Model A for HD 37124 is interesting in two other respects. It contains three planets in nearly circular orbits, the first such triple-planet system. The other three triple-planet systems have sizeable orbital eccentricities. Moreover, model A is composed of three planets that have nearly the same minimum mass, $M \sin i \approx 0.65 M_{\mathrm{J}}$. Indeed, we suspect that significant constraints can be placed on the inclination of this system (or mutual inclinations) by demanding that it be stable. Significant inclinations would render the planets more massive than their values of $M \sin i$, causing a dynamical instability.

Progress in Doppler precision and in dynamical analyses is leading to a new capability in the study of exoplanets. Systems remaining to be discovered tend to have low planetary masses, yielding small velocity amplitudes relative to Doppler errors. The weak signals make it difficult to establish both the minimum number of planets required in the model and the orbital parameters with high precision. A combination of precise velocities and dynamical simulations offers a path toward assessing the various models by imposing long-term stability as a requirement. Such approaches constrain the number of planets and their orbital parameters and imply plausible formation scenarios. The HD 37124 system has a wide variety of plausible Keplerian solutions but only two domains that are dynamically viable.

The common occurrence of mean motion and secular resonances suggests that migration of planets within the protoplanetary disk must be common. We know of no better evidence of the migration process than this plethora of resonances. The initial orbits, migration speeds, and planet masses must play significant roles in the final detailed resonance of the system.

In contrast, the hierarchical planetary systems, such as HD 12661 with its two planets having periods of 260 and 1400 days and HD 38529 with periods of 14.3 days and $6.0 \mathrm{yr}$, show that some systems avoid resonance trapping. The two-component system HD 217107 with periods of 7.1 and $>2500$ days is certainly a new hierarchical case. These nonresonant systems suggest that the planets formed far from each other within the 
disk, preventing migration from trapping them. More intriguing are the near-resonance systems, such as HD 108874 and $v$ And, which are apparently interacting but not obviously in a resonance. One wonders what physical scenario brings two planets into an interacting state but never deeply trapped in a resonance.

More work is required to ascertain any resonances in HD 108874 and HD 37124. More theoretical attention to the nearresonant systems would be valuable. The near $4: 1$ resonance of HD 108874 is reminiscent of the familiar Jupiter-Saturn pair, whose dynamics are affected by the $5: 2$ near-commensurability (the so-called Great Inequality), but which nevertheless exhibits circulation of all of the relevant resonant arguments.

Probably related is that many single planets reside in highly eccentric orbits, without any massive planets or stars currently orbiting the star. The stars 14 Her and 70 Vir are examples of single (and massive) exoplanets that reside in eccentric orbits, but the velocities show no evidence of additional companions, planetary or stellar. Apparently, high eccentricities occur without benefit of any current companions, an attribute of observed exoplanets that is not well explained. Models of disk pumping of eccentricities (Goldreich \& Sari 2003) require more attention (Schäfer et al. 2004). The similarity in the eccentricities of single planets and multiple planets suggests that the origin of the eccentricities may be the same. Such a similar mechanism is re- markable because the planets in multiplanet systems are definitely perturbed by each other while single planets enjoy no such interaction currently.

We thank A. Cumming, E. Ford, and Kevin Apps for valuable discussions. We thank Mike Liu for adaptive optics images of target stars. We gratefully acknowledge the efforts and dedication of the Keck Observatory staff. We also thank Lou Boyd for his ongoing support of Fairborn Observatory. We appreciate support by NASA grant NAG5-75005 and by NSF grants AST 03-07493 and AST 99-88087 (to S. S. V.), by NASA grant NAG5-12182, and travel support from the Carnegie Institution of Washington (to R. P. B.). G. W. H. acknowledges support from NASA grant NCC5-511 and NSF grant HRD-9706268. We are also grateful for support by Sun Microsystems. We thank the NASA and UC Telescope assignment committees for allocations of telescope time. This research has made use of the SIMBAD database, operated at CDS, Strasbourg, France. The authors wish to extend special thanks to those of Hawaiian ancestry on whose sacred mountain of Mauna Kea we are privileged to be guests. Without their generous hospitality, the Keck observations presented herein would not have been possible.

\section{REFERENCES}

Alibert, Y., Mordasini, C., Benz, W., \& Winisdoerffer, C. 2005, A\&A, 434, 343 Allende Prieto, C., \& Lambert, D. L. 1999, VizieR Online Data Catalog, 335, 20555

Armitage, P. J., Clarke, C. J., \& Palla, F. 2003, MNRAS, 342, 1139

Bodenheimer, P., Laughlin, G., \& Lin, D. N. C. 2003, ApJ, 592, 555

Brown, R. A. 2004, ApJ, 610, 1079

Bryden, G., Różyczka, M., Lin, D. N. C., \& Bodenheimer, P. 2000, ApJ, 540, 1091

Butler, R. P. 1993, Ph.D. thesis, Maryland Univ.

Butler, R. P., \& Marcy, G. W. 1996, ApJ, 464, L153

Butler, R. P., Marcy, G. W., Fischer, D. A., Brown, T. M., Contos, A. R., Korzennik, S. G., Nisenson, P., \& Noyes, R. W. 1999, ApJ, 526, 916

Butler, R. P., Marcy, G. W., Vogt, S. S., Fischer, D. A., Henry, G. W., Laughlin, G., \& Wright, J. T. 2003, ApJ, 582, 455

Butler, R. P., Marcy, G. W., Williams, E., McCarthy, C., Dosanjh, P., \& Vogt, S. S. 1996, PASP, 108, 500

Chiang, E. I., \& Murray, N. 2002, ApJ, 576, 473

Chiang, E. I., Tabachnik, S., \& Tremaine, S. 2001, AJ, 122, 1607

Cumming, A., Marcy, G. W., \& Butler, R. P. 1999, ApJ, 526, 890

D’Angelo, G., Kley, W., \& Henning, T. 2003, ApJ, 586, 540

Eaton, J. A., Henry, G. W., \& Fekel, F. C. 2003, in The Future of Small Telescopes in the New Millennium, Vol. 2: The Telescopes We Use, ed. T. D. Oswalt (Dordrecht: Kluwer), 189

ESA. 1997, VizieR Online Data Catalog, 1239

Fernandes, J., \& Santos, N. C. 2004, A\&A, 427, 607

Fischer, D. A., Marcy, G. W., Butler, R. P., Vogt, S. S., \& Apps, K. 1999, PASP, 111,50

Fischer, D. A., Marcy, G. W., Butler, R. P., Vogt, S. S., Frink, S., \& Apps, K. 2001, ApJ, 551, 1107

Fischer, D. A., \& Valenti, J. 2005, ApJ, 622, 1102

Ford, E. B. 2004, in AIP Conf. Proc. 713, The Search for Other Worlds, ed. S. S. Holt \& D. Deming (New York: AIP), 27

Ford, E. B., Rasio, F. A., \& Yu, K. 2003, in ASP Conf. Ser. 294, Scientific Frontiers in Research on Extrasolar Planets, ed. D. Deming \& S. Seager (San Francisco: ASP), 181

Girardi, L., Bertelli, G., Bressan, A., Chiosi, C., Groenewegen, M. A. T., Marigo, P., Salasnich, B., \& Weiss, A. 2002, A\&A, 391, 195

Goldreich, P., \& Sari, R. 2003, ApJ, 585, 1024

Goździewski, K. 2003, A\&A, 398, 315

Henry, G. W. 1995a, in ASP Conf. Ser. 79, Robotic Telescopes: Current Capabilities, Present Developments, and Future Prospects for Automated Astronomy, ed. G. W. Henry \& J. A. Eaton (San Francisco: ASP), 37

. 1995b, in ASP Conf. Ser. 79, Robotic Telescopes: Current Capabilities, Present Developments, and Future Prospects for Automated Astronomy, ed. G. W. Henry \& J. A. Eaton (San Francisco: ASP), 44
Henry, G. W. 1999, PASP, 111, 845

Henry, G. W., Baliunas, S. L., Donahue, R. A., Fekel, F. C., \& Soon, W. 2000a, ApJ, 531, 415

Henry, G. W., Marcy, G. W., Butler, R. P., \& Vogt, S. S. 2000b, ApJ, 529, L41

Henry, T. J., Soderblom, D. R., Donahue, R. A., \& Baliunas, S. L. 1996, AJ, 111,439

Ida, S., \& Lin, D. N. C. 2004, ApJ, 604, 388

Kerr, R. 2002, Science, 296, 2124

Laughlin, G., Butler, R. P., Fischer, D. A., Marcy, G., Wolf, A., \& Vogt, S. S. 2005, ApJ, 622, 1182

Laughlin, G., \& Chambers, J. E. 2001, ApJ, 551, L109

Laws, C., Gonzalez, G., Walker, K. M., Tyagi, S., Dodsworth, J., Snider, K., \& Suntzeff, N. B. 2003, AJ, 125, 2664

Lee, M. H., \& Peale, S. J. 2002, ApJ, 567, 596

Levison, H. F., Lissauer, J. J., \& Duncan, M. J. 1998, AJ, 116, 1998

Lissauer, J. J. 1995, Icarus, 114, 217

Lissauer, J. J., \& Rivera, E. J. 2001, ApJ, 554, 1141

Marcy, G. W., \& Butler, R. P. 1992, PASP, 104, 270

. 1996, ApJ, 464, L147

Marcy, G. W., Butler, R. P., Fischer, D. A., \& Vogt, S. S. 2004, in ASP Conf. Ser. 321, Extrasolar Planets: Today and Tomorrow, ed. J.-P. Beaulieu, A. Lecavelier des Etangs, \& C. Terquem (San Francisco: ASP), 3

Marcy, G. W., Butler, R. P., Vogt, S. S., Fischer, D. A., Henry, G. W., Laughlin, G., Wright, J. T., \& Johnson, J. A. 2005, ApJ, 619, 570

Marzari, F., \& Weidenschilling, S. J. 2002, Icarus, 156, 570

Mayor, M., \& Queloz, D. 1995, Nature, 378, 355

Mayor, M., Udry, S., Naef, D., Pepe, F., Queloz, D., Santos, N. C., \& Burnet, M. 2004, A\&A, 415, 391

Murray, C. D., \& Dermott, S. F. 1999, Solar System Dynamics (Princeton: Princeton Univ. Press)

Naef, D., et al. 2003, A\&A, 410, 1051

Nauenberg, M. 2002, ApJ, 568, 369

Nelson, R. P., \& Papaloizou, J. C. B. 2002, MNRAS, 333, L26

Noyes, R. W., Hartmann, L. W., Baliunas, S. L., Duncan, D. K., \& Vaughan, A. H. 1984, ApJ, 279, 763

Paulson, D. B., Saar, S. H., Cochran, W. D., \& Henry, G. W. 2004, AJ, 127, 1644 Queloz, D., et al. 2001, A\&A, 379, 279

Rivera, E. J., \& Lissauer, J. J. 2000, ApJ, 530, 454 2001, ApJ, 558, 392

Saar, S. H., Butler, R. P., \& Marcy, G. W. 1998, ApJ, 498, L153

Santos, N. C., Israelian, G., \& Mayor, M. 2004, A\&A, 415, 1153

Santos, N. C., Mayor, M., Naef, D., Pepe, F., Queloz, D., Udry, S., \& Blecha, A. 2000a, A\&A, 361, 265

Santos, N. C., Mayor, M., Naef, D., Pepe, F., Queloz, D., Udry, S., Burnet, M., \& Revaz, Y. 2000b, A\&A, 356, 599 
Santos, N. C., et al. 2003, A\&A, 406, 373

Schäfer, C., Speith, R., Hipp, M., \& Kley, W. 2004, A\&A, 418, 325

Seagroves, S., Harker, J., Laughlin, G., Lacy, J., \& Castellano, T. 2003, PASP, 115,1355

Shao, M., ed. 2003, Interferometry in Space (Proc. SPIE Vol. 4852)

Strassmeier, K., Washuettl, A., Granzer, T., Scheck, M., \& Weber, M. 2000, A\&AS, 142,275

Thommes, E. W., \& Lissauer, J. J. 2003, ApJ, 597, 566

Trauger, J., et al. 2003, BAAS, 203, 03.03

Trilling, D. E., Lunine, J. I., \& Benz, W. 2002, A\&A, 394, 241

Udry, S., Mayor, M., \& Queloz, D. 2003, in ASP Conf. Ser. 294, Scientific Frontiers in Research on Extrasolar Planets, ed. D. Deming \& S. Seager (San Francisco: ASP), 17
Valenti, J. A., \& Fischer, D. A. 2005, ApJS, 159, 141

Vaníček, P. 1971, Ap\&SS, 12, 10

Vogt, S. S. 1987, PASP, 99, 1214

Vogt, S. S., Marcy, G. W., Butler, R. P., \& Apps, K. 2000, ApJ, 536, 902

Vogt, S. S., et al. 1994, Proc. SPIE, 2198, 362

Wright, J. T. 2004, AJ, 128, 1273

Wright, J. T., Henry, G. W., Marcy, G. W., Butler, R. P., \& Vogt, S. S. 2005, PASP, 117, 657

Wright, J. T., Marcy, G. W., Butler, R. P., \& Vogt, S. S. 2004, ApJS, 152, 261 